\title{
TIME-DELAY REGULARIZATION OF ANISOTROPIC DIFFUSION AND IMAGE PROCESSING
}

\author{
Abdelmounim Belahmidi ${ }^{1}$ And Antonin Chambolle ${ }^{2}$
}

\begin{abstract}
We study a time-delay regularization of the anisotropic diffusion model for image denoising of Perona and Malik [IEEE Trans. Pattern Anal. Mach. Intell 12 (1990) 629-639], which has been proposed by Nitzberg and Shiota [IEEE Trans. Pattern Anal. Mach. Intell 14 (1998) 826-835]. In the two-dimensional case, we show the convergence of a numerical approximation and the existence of a weak solution. Finally, we show some experiments on images.
\end{abstract}

Mathematics Subject Classification. 68U10, 35K55, 35M10.

Received: March 2, 2004.

\section{INTRODUCTION}

In a well-known paper, Perona and Malik [18] have proposed a model for image restoration based on the following partial differential equation:

$$
\frac{\partial u}{\partial t}=\operatorname{div}\left(g\left(|D u|^{2}\right) D u\right) \quad u(\cdot, 0)=u_{0} .
$$

Here $u_{0}$ is the grey level intensity of the original image, $u(\cdot, t)$ is the restored version, that depends on the scale parameter $t$, and $g$ is a smooth non-increasing positive function with $g(0)=1$ and $s g\left(s^{2}\right) \rightarrow 0$ at infinity. The main idea is that the restoration process obtained by the equation is conditional: if $x$ is an edge point, where the gradient is large, then the diffusion will be stopped and therefore the edge will be kept. If $x$ is in a homogeneous area, the gradient has to be small, and the diffusion will tend to smooth around $x$. By introducing an edge stopping function $g\left(|D u|^{2}\right)$ in the diffusion process, the model has been considered as an important improvement of the theory of edge detection [15]. The experiments of Perona and Malik were very impressive, edges remained stable over a very long time. In their paper [18], they claim that edge detection based on this process clearly outperforms the Canny edge detector [3].

Unfortunately, the Perona-Malik model is ill-posed. Indeed, among the functions which Perona and Malik advocate in their papers, we find $g\left(s^{2}\right)=1 /\left(1+s^{2}\right)$ or $g\left(s^{2}\right)=\mathrm{e}^{-s^{2}}$ for which no correct theory of equation (1)

\footnotetext{
Keywords and phrases. Image restoration, edge detection, Perona-Malik equation, time-delay regularization.

1 CEREMADE (CNRS UMR 7534), Université de Paris Dauphine, 75775 Paris Cedex 16, France.

belamid@ceremade.dauphine.fr

2 CMAP (CNRS UMR 7641), École Polytechnique, 91128 Palaiseau Cedex, France. antonin.chambolle@polytechnique.fr
} 
is available. By writing the equation in dimension two:

$$
\frac{\partial u}{\partial t}=g\left(|D u|^{2}\right)|D u| \operatorname{div}\left(\frac{D u}{|D u|}\right)+\left(g\left(|D u|^{2}\right)+2|D u|^{2} g^{\prime}\left(|D u|^{2}\right)\right) D^{2} u\left(\frac{D u}{|D u|}, \frac{D u}{|D u|}\right),
$$

where $D^{2} u\left(\frac{D u}{|D u|}, \frac{D u}{|D u|}\right)$ is the second derivative of $u$ in the gradient direction and $|D u| \operatorname{div}\left(\frac{D u}{|D u|}\right)$ is the second derivative in the orthogonal direction, we observe that the diffusion runs backwards if $s g\left(s^{2}\right)$ is non-increasing. Then, in the regions where the gradient of a solution is large, the process can be interpreted as a backwards heat equation which is actually ill-posed. In the continuous setting, it means that (1) may have no solution at all. One could also imagine very close pictures producing divergent solutions [11]. In practice, the equation is discretized into a (obviously well-posed) finite-dimensional version of (1), however, it does not seem correct to interpret such a discretization as an approximation of the ill-posed problem (1).

For these reasons, there have been many attempts to understand the Perona-Malik equation and find out whether (1) can be given a sound interpretation. There are essentially two approaches: the first, motivated by favorable numerical results, consists in studying the original equation and in establishing theoretical results that explain the observed behaviour. The second approach consists in modifying the equation by regularizing the term $g\left(|D u|^{2}\right)$ in order to get a well-posed equation.

\section{The Perona and Malik equation and the Regularized versions}

First, we expose the main mathematical results established on the Perona-Malik model. Most of these results are restricted to the dimension one; the unique result in dimension two, given by You et al. [20] confirms the ill-posedness of the equation. Kawohl and Kutev [13] establish, in 1D, nonexistence of global weak solution, and prove the existence and uniqueness of a classical solution only if the initial data has everywhere a small slope. In this case the equation remains parabolic for all time and there is no edge to preserve: the diffusion smooths the data, like the heat equation would do. They also prove a comparison principle under special assumptions on the initial data.

Kichenassamy [14] shows that in general the Perona-Malik equation does not have a weak solution if the initial data is not analytic in a neighborhood of high gradient regions. His argument is based on interior regularity properties of parabolic equations. Only in dimension one, he proposes a notion of generalized solutions, which are piecewise linear with jumps, and shows existence.

Adopting a numerical viewpoint, Esedoglu [7] studies the one-dimensional Perona-Malik scheme. He establishes by a scaling argument the convergence to an evolution in the continuous setting. The resulting evolution solves a system of heat equations coupled to each other through nonlinear boundary conditions.

Working in dimension one clearly reduces the difficulty by eliminating the first term of (2) which is nothing but the mean curvature motion operator with the coefficient $g\left(|D u|^{2}\right)$. As it is known, the mean curvature motion evolves each level line $\{u=C\}$ with a normal speed proportional to its curvature (see $[1,8]$ for more details).

In dimension two, You et al. [20] express the anisotropic diffusion of Perona and Malik as the steepest descent of an energy surface and analyze the behaviour of the model. They prove that the ill-posedness is caused by the fact that the energy functional has an infinite number of global minima that are dense in the image space. Each of these minima corresponds to a piecewise constant image. This means that slightly different initial images may end up in different minima for large $t$.

As mentioned, another approach relies on the idea that the ill-posedness may be alleviated through the introduction of a smooth version of $g\left(|D u|^{2}\right)$. There are essentially two propositions which we consider as a direct derivation from the Perona-Malik Model. The first consists in a spatial regularization, as in the following model:

$$
\frac{\partial u}{\partial t}=\operatorname{div}\left(g\left(\left|D G_{\sigma} * u\right|^{2}\right) D u\right),
$$


whereby $g\left(|D u|^{2}\right)$ is replaced by $g\left(\left|D G_{\sigma} * u\right|^{2}\right)$, where $G_{\sigma}$ is a Gaussian with variance $\sigma$. In [4], Catté $e t a l$. prove existence, uniqueness and regularity of a solution. It is known that $G_{\sigma} * u(x, t)$ is nothing but the solution at scale $\sigma$ of the heat equation with $u(x, t)$ as initial data.

A first observation is that near a sharp corner, the diffusion coefficient $g\left(\left|D G_{\sigma} * u\right|^{2}\right)$ may remain very large, hence this model will be unable to preserve corners.

Another problem is the choice of the regularization parameter $\sigma$. In fact, this choice is critical in the sense that the diffusion process would be ill-posed if $\sigma=0$, while image features would be blurred for too large an $\sigma$. As proposed by Whitaker and Pizer [19], the regularization parameter $\sigma$ should be a decreasing function in $t$, by using large $\sigma$ initially to suppress noise and reducing $\sigma$ so that image features are not further blurred. In spite of this, the choice of the initial and final values of $\sigma$ remains an open question.

The second proposition is a time-delay regularization, where one replaces $|D u|^{2}$ with an average of its values from 0 to $t$. Then $g\left(|D u|^{2}\right)$ is replaced with $g(v)$ with:

$$
v(x, t)=\mathrm{e}^{-t} v_{0}(x)+\int_{0}^{t} \mathrm{e}^{s-t}|D u(x, s)|^{2} \mathrm{~d} s,
$$

where $v_{0}$ is an initial data, for example $v_{0}=0$ or $\left|D u_{0}\right|^{2}$. Therefore the new diffusion process is described by the following system:

$$
\begin{array}{ll}
\frac{\partial u}{\partial t}=\operatorname{div}(g(v) D u) & u(\cdot, 0)=u_{0} \\
\frac{\partial v}{\partial t}=|D u|^{2}-v & v(\cdot, 0)=v_{0} .
\end{array}
$$

Proposed by Nitzberg and Shiota [17], this model is very close to the Perona-Malik equation since there is no spatial smoothing. In particular, it should mean that there is no previous motion of the features in the diffusion process. In [2] the authors of the present paper have shown that in any dimension, the system (5)-(6) admits a unique classical solution $(u, v)$ which can blow up in finite time, and that as long as the solution exists, the equation satisfies the maximum principle and does not create spurious information (that is, strict local extrema). These properties of the system (5)-(6) have encouraged us to study it from a numerical viewpoint. Let us mention that a similar equation involving the time-delay regularization of an anisotropic diffusion tensor has been already studied, also for image processing, by Cottet and El Ayyadi [6].

This paper is organized as follows: in Section 2 we propose a natural discretization in time of (5)-(6) with $|D u|^{2}$ replaced by $F\left(|D u|^{2}\right), F$ being a sort of truncation. In practice, this modification does not have any impact on the output images since the threshold implicitly exists in the numerical scheme. Indeed, if the discrete scheme satisfies the maximum principle, then the discrete gradient is always bounded (for example by $\left(\max u_{0}-\min u_{0}\right) / \Delta x, \Delta x$ being the grid size). Theoretically, the introduction of $F$ is a huge regularization of the system (we will see that it yields existence of a weak solution for all time). Section 3 proposes a numerical scheme for solving the system, and Section 4 shows some experiments on synthetic and natural images. In Section 5 we establish a priori estimates and regularity results on the proposed approximation and prove the main result of this paper. In Section 6 we give the proofs of two technical results on elliptic equations that are needed in Section 5.

\section{Numerical APPROXimation}

The goal of this paper is to study and approximate numerically the system:

$$
\begin{array}{ll}
\frac{\partial u}{\partial t}=\operatorname{div}(g(v) D u) & u(\cdot, 0)=u_{0}, \\
\frac{\partial v}{\partial t}=F\left(|D u|^{2}\right)-v & v(\cdot, 0)=v_{0},
\end{array}
$$


in $\Omega \times(0, T)$ where $\Omega=(0,1)^{2}, 0<T<\infty$. We will show that the system admits a weak solution, under the following technical assumptions:

$-g \in C^{1}([0,+\infty))$ is a positive non-increasing function with $g(0)=1$ and $g(+\infty)=0$;

- $F \in C^{1}([0,+\infty))$ is a smooth version of $s \rightarrow \min (s, \mathrm{M})$, where $\mathrm{M}>0$ is a (large) real number (in particular, we assume $\left.0 \leq F^{\prime} \leq 1\right)$.

Fixed $\delta t>0$, we define the sequence $\left(u_{\delta t}^{n}, v_{\delta t}^{n}\right)_{n}$ by the semi-implicit scheme:

$$
\begin{aligned}
\left(u_{\delta t}^{0}, v_{\delta t}^{0}\right)= & \left(u_{0}, v_{0}\right) \in\left(H^{1}(\Omega) \cap L^{\infty}(\Omega)\right) \times\left(H^{1}(\Omega) \cap L^{\infty}(\Omega)\right), v_{0} \geq 0 \text { and } \\
& \frac{u_{\delta t}^{n+1}-u_{\delta t}^{n}}{\delta t}=\left.\operatorname{div}\left(g\left(v_{\delta t}^{n}\right) D u_{\delta t}^{n+1}\right) \quad \frac{\partial u_{\delta t}^{n+1}}{\partial n}\right|_{\partial \Omega}=0 \\
& \frac{v_{\delta t}^{n+1}-v_{\delta t}^{n}}{\delta t}=F\left(\left|D u_{\delta t}^{n+1}\right|^{2}\right)-v_{\delta t}^{n+1} .
\end{aligned}
$$

We define the piecewise constant (in $t>0$ ), functions

$$
u_{\delta t}(x, t)=u_{\delta t}^{[t / \delta t]+1}(x)
$$

where [.] denotes the integer part. We also define $\left(v_{\delta t}\right)$ in the same way. Then we can write the discrete system (9)-(10) in the form $\left(\tau^{-\delta t}\right.$ is defined by $\left.\tau^{-\delta t} f(\cdot, t)=f(\cdot, t-\delta t)\right)$ :

$$
\begin{aligned}
& \frac{u_{\delta t}-\tau^{-\delta t} u_{\delta t}}{\delta t}=\operatorname{div}\left(g\left(\tau^{-\delta t} v_{\delta t}\right) D u_{\delta t}\right),\left.\quad \frac{\partial u_{\delta t}}{\partial n}\right|_{\partial \Omega}=0, \\
& \frac{v_{\delta t}-\tau^{-\delta t} v_{\delta t}}{\delta t}=F\left(\left|D u_{\delta t}\right|^{2}\right)-v_{\delta t} .
\end{aligned}
$$

The main result of this paper is the following theorem:

Theorem 1. Let $T>0$. There exist a subsequence $\left(u_{\delta t_{j}}, v_{\delta t_{j}}\right)$ of $\left(u_{\delta t}, v_{\delta t}\right)$ and $(u, v)$ a weak solution of the system (7)-(8) in $\left(H^{1}(\Omega \times(0, T)) \cap L^{\infty}(\Omega \times(0, T))\right) \times\left(H^{1}(\Omega \times(0, T)) \cap L^{\infty}(\Omega \times(0, T))\right)$ such that, we have the convergences, as $j \rightarrow+\infty$ :

$$
\begin{array}{ll}
u_{\delta t_{j}} \longrightarrow u & \text { strongly in } L^{2}\left(0, T ; H^{1}(\Omega)\right), \\
v_{\delta t_{j}} \longrightarrow v & \text { weakly in } L^{2}\left(0, T ; H^{1}(\Omega)\right) .
\end{array}
$$

The proof of this theorem will be given in Section 5 .

\section{Discretization}

To discretize (9)-(10) we denote by $u_{i, j}^{n}$ (resp. $v_{i, j}^{n}$ ) the approximation of $u$ (resp. $v$ ) at point $(i h, j h)$ $(0 \leq i, j \leq N)$ and time $t=n \delta t$, where the size of the initial image $u_{0}$ is given by $N \times N$ and $h=1 / N$. Using the following finite-differences formulas:

$$
\begin{array}{cc}
\Delta_{+}^{x} w=w_{i+1, j}-w_{i, j}, & \Delta_{-}^{x} w=w_{i, j}-w_{i-1, j}, \\
\Delta_{+}^{y} w=w_{i, j+1}-w_{i, j} \text { and } & \Delta_{-}^{y} w=w_{i, j}-w_{i, j-1},
\end{array}
$$

the approximation of $\operatorname{div}(g(v) D u)$ at point $(i h, j h)$ and at scale $t=(n+1) \delta t$ is given by:

$$
\frac{1}{h^{2}}\left(\Delta_{-}^{x}\left(g\left(v_{i, j}^{n}\right) \Delta_{+}^{x} u_{i, j}^{n+1}\right)+\Delta_{-}^{y}\left(g\left(v_{i, j}^{n}\right) \Delta_{+}^{y} u_{i, j}^{n+1}\right)\right) .
$$


Then the equation (9) becomes:

$$
\begin{aligned}
\frac{u_{i, j}^{n+1}-u_{i, j}^{n}}{\delta t}= & \frac{1}{h^{2}}\left\{g\left(v_{i, j}^{n}\right)\left(u_{i+1, j}^{n+1}-u_{i, j}^{n+1}\right)-g\left(v_{i-1, j}^{n}\right)\left(u_{i, j}^{n+1}-u_{i-1, j}^{n+1}\right)\right. \\
& \left.+g\left(v_{i, j}^{n}\right)\left(u_{i, j+1}^{n+1}-u_{i, j}^{n+1}\right)+g\left(v_{i, j-1}^{n}\right)\left(u_{i, j}^{n+1}-u_{i, j-1}^{n+1}\right)\right\}
\end{aligned}
$$

with the Neumann boundary condition:

$$
\begin{aligned}
& u_{i, 0}^{n+1}-u_{i, 1}^{n+1}=0, \quad u_{i, N-1}^{n+1}-u_{i, N}^{n+1}=0, \quad \text { for } \quad 0 \leq i \leq N, \\
& u_{0, j}^{n+1}-u_{1, j}^{n+1}=0, \quad u_{N-1, j}^{n+1}-u_{N, j}^{n+1}=0, \quad \text { for } \quad 0 \leq j \leq N .
\end{aligned}
$$

Rearranging the right hand side of (15), we get

$$
\frac{u^{n+1}-u^{n}}{\delta t}+h^{-2} A\left(v^{n}\right) u^{n+1}=0
$$

where the matrix $A\left(v^{n}\right)$ is tridiagonal by blocks, and positive defined. By classical arguments [5] we know that $\left[I+\delta t h^{-2} A\left(v^{n}\right)\right]$ is invertible.

To avoid any additional anisotropy in the scheme, we try to build a discrete gradient of $u$ in (10) as rotationally invariant as possible. We use the discretization proposed in $[4,17]$ which writes:

$$
\begin{aligned}
& \Delta^{x} w=\left(1+2^{\frac{1}{2}}\right)^{-1}\left\{\left(w_{i+1, j}-w_{i-1, j}\right)+2^{-\frac{1}{2}}\left(w_{i+1, j-1}-w_{i-1, j-1}\right)+2^{-\frac{1}{2}}\left(w_{i+1, j+1}-w_{i-1, j+1}\right)\right\} \\
& \Delta^{y} w=\left(1+2^{\frac{1}{2}}\right)^{-1}\left\{\left(w_{i, j+1}-w_{i, j-1}\right)+2^{-\frac{1}{2}}\left(w_{i+1, j+1}-w_{i+1, j-1}\right)+2^{-\frac{1}{2}}\left(w_{i-1, j+1}-w_{i+1, j-1}\right)\right\}
\end{aligned}
$$

The discretization of (10) is then written (assuming that in the whole range $\left[0, \max \left(\left(\Delta^{x} u\right)^{2}+\left(\Delta^{y} u\right)^{2}\right)\right]$, we have $F(s)=s$ )

$$
v_{i, j}^{n+1}=\frac{1}{1+\delta t}\left(\delta t h^{-2}\left(\left(\Delta^{x} u\right)^{2}+\left(\Delta^{y} u\right)^{2}\right)+v_{i, j}^{n}\right) .
$$

We can now give a discrete version of the maximum principle and show that the proposed algorithm will not create new information (local extrema).

Lemma 1. For all $n>0$ and $(k, l), 0 \leq k, l \leq N$, we have:

$$
\min _{i, j} u_{i, j}^{0} \leq \ldots \leq \min _{i, j} u_{i, j}^{n} \leq u_{k, l}^{n+1} \leq \max _{i, j} u_{i, j}^{n} \leq \cdots \leq \max _{i, j} u_{i, j}^{0} .
$$

In particular, if $u_{k, l}^{n+1}$ is a strict local maximum (resp. strict local minimum) of $\left(u_{i, j}^{n+1}\right)$ then

$$
u_{k, l}^{n+1}<u_{k, l}^{n} \quad\left(\text { resp. } \quad u_{k, l}^{n+1}>u_{k, l}^{n}\right)
$$

Proof. Let $u_{k, l}^{n+1}$ a global maximum of $\left(u_{i, j}^{n+1}\right)$, then in particular:

$$
\begin{array}{ll}
u_{k, l}^{n+1}-u_{k+1, l}^{n+1} \geq 0, & u_{k, l}^{n+1}-u_{k-1, l}^{n+1} \geq 0, \\
u_{k, l}^{n+1}-u_{k, l+1}^{n+1} \geq 0, & u_{k, l}^{n+1}-u_{k, l-1}^{n+1} \geq 0 .
\end{array}
$$

Using (15), and the fact that $g>0$, we obtain:

$$
u_{k, l}^{n+1} \leq u_{k, l}^{n}
$$


and we deduce:

$$
\max _{i, j} u_{i, j}^{n+1} \leq \max _{i, j} u_{i, j}^{n} \leq \cdots \leq \max _{i, j} u_{i, j}^{0} .
$$

In the same way we prove the "min" part of (17), by considering $u_{k, l}^{n+1}$ a global minimum of $\left(u_{i, j}^{n+1}\right)$. We prove (18) by using the same argument and the fact that we now have strict inequalities in (19).

\section{EXPERIMENTS}

In Figure 1 (resp. Figs. 2 and 3) we compare the performances of our scheme with the Catté et al. model [4] (resp. with the Perona-Malik model [18]) and in Figure 4 we present an example of restoration on a natural image. The experiments have been done with the edge stopping function

$$
g\left(s^{2}\right)=\frac{1}{1+\left(s^{2} / \lambda^{2}\right)} .
$$

We have chosen a value of $\lambda=6$, for images in the range $[0,255]$ and with a spatial grid size $h=1$ (contrarily to the convention in the previous section). The temporal increment we have used is $\delta t=0.1$.

Figure 1a is a synthetic image $(128 \times 128)$ representing superimposed shapes having each one a constant grey level. Figure $1 \mathrm{~b}$ shows image 1a where $20 \%$ of Gaussian noise is added. We represent by Figure 1c the restoration of the noisy image with the Catté et al. model (3) at scales 4 and 8 (from left to right) and by Figure $1 \mathrm{~d}$ the restoration with our scheme at the same scales. Notice that respectively, the scales 4 and 8 correspond to the stopping time $t=8$ and 32. As explained in [4] by the authors of the model (3), the scale $\sigma$ used in the convolution term $G_{\sigma} * u$ must be taken in relation to the stopping time. Thus in Figure 1c we have used $\sigma=4$ and 8 .

As mentioned in Section 1 the threshold introduced by $F$ implicitly exists in the numerical scheme. Indeed, since the discrete scheme satisfies the maximum principle and the fact that spatial increment is assumed to be 1 , then the discrete gradient is always bounded by $\sqrt{2}\left(\max u_{0}-\min u_{0}\right)$ and $\mathrm{M}$ can be chosen to be $2\left(\max u_{0}-\right.$ $\left.\min u_{0}\right)^{2}$.

In the left image of $1 \mathrm{~b}$, the noise is smoothed in the homogeneous areas but is kept near the edges. This drawback is caused by the fact that the diffusion is inhibited also in the neighborhood of edges. Whereas in the left image of $1 \mathrm{c}$, the noise is only partially smoothed but in a uniform way. In the right image of $1 \mathrm{~b}$ the edges and corners are blurred. Indeed, we know that $\left\|D G_{\sigma} * u\right\|_{L^{\infty}}$ decreases for large values of $\sigma$ consequently for large values of $\sigma$ we diffuse more near edges: in particular, if $\left\|D G_{\sigma} * u\right\|_{L^{\infty}}<\lambda$, the diffusion is never inhibited. Whereas in the right image of $1 \mathrm{c}$, the noise has disappeared and the reconstructed image is very close to the original.

The goal of Figures 2 and 3 is to compare the performance of our scheme with the Perona-Malik model in regions where the gradient is moderately large, such as affine and blurred regions. To this aim, we have constructed a synthetic images, 2a and 3a where $20 \%$ of Gaussian noise is added, in which we have superimposed a blurred disk, a constant disk, and a disk with smoothly varying intensity. The resulting images, $2 \mathrm{~b}$ with the Perona-Malik model and 2c with our scheme, present a staircasing effect and are perceptually very close. The same remarks holds in the case where the noise is added, images $3 \mathrm{~b}$ and $3 \mathrm{c}$.

In the experiences $2 \mathrm{~d}$ and $3 \mathrm{~d}$ we have introduced a relaxation parameter $w$ in the second equation: $\left(v_{\delta t}^{n+1}-\right.$ $\left.v_{\delta t}^{n}\right) / \delta t=w\left(F\left(\left|D u_{\delta t}^{n+1}\right|^{2}\right)-v_{\delta t}^{n+1}\right)$ in order to delay as far as possible the staircasing effect, by choosing a small value of $w$. Here we use $w=10^{-2}$. Note that in these figures, all diffusions are be done at scale 20 which corresponds to the stopping time $t=200$.

Figure 4 right represents a natural image $(256 \times 256)$ without additive noise and Figure 4 left represents its restoration with our scheme at scale 5 that corresponds to the stopping time $t=12.5$. We remark that salient edges and textures are preserved (see for example the top of the hat) whereas the noise in homogeneous areas is smoothed. 

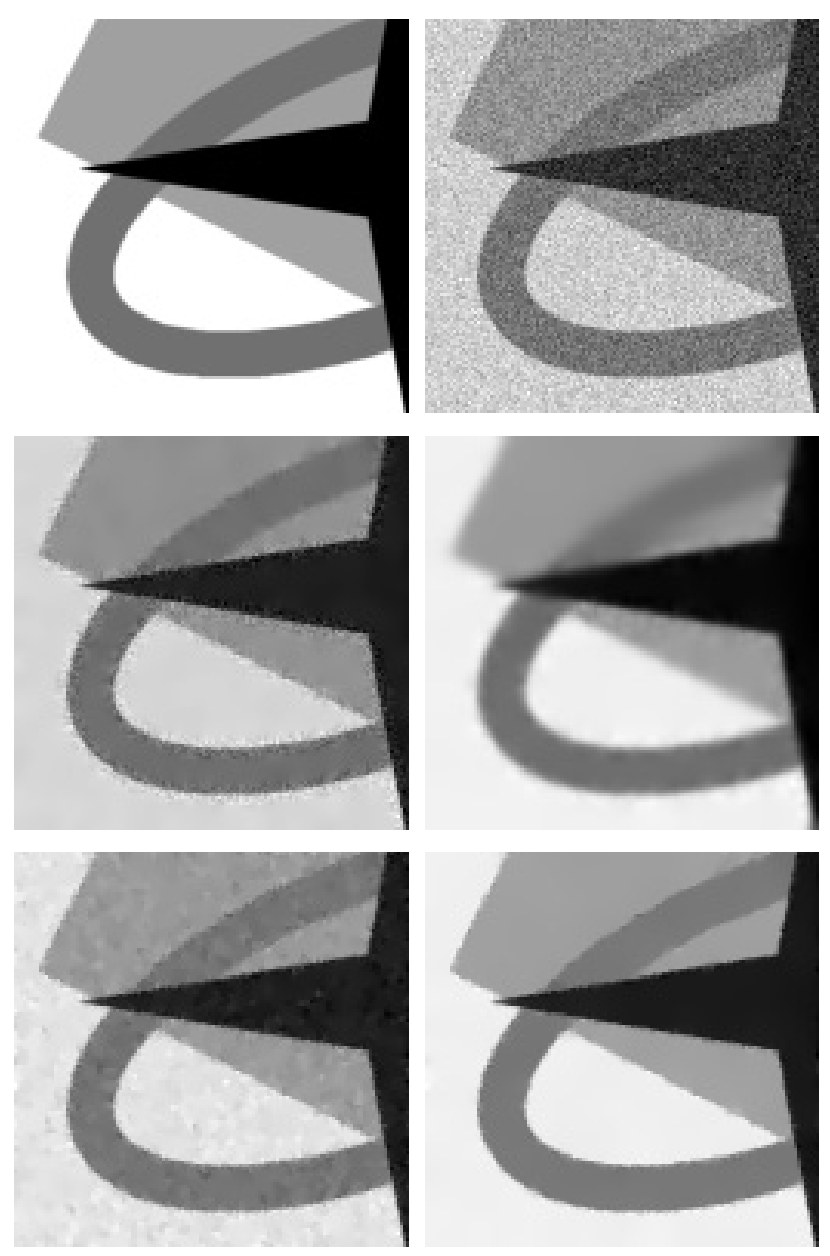

Figure 1. Top left: (a) Original image. Top right: (b) Image (a) with $20 \%$ of Gaussian noise. Middle line: (c) Image (b) restored by the Catté et al. model [4] with scales 4, and 8. Bottom line: (d) Image (b) restored by our scheme with scales 4 , and 8.

\section{Numerical ANALYSIS}

First we check that our scheme makes sense. Indeed, for all $\delta t>0$, the sequence $\left(u_{\delta t}^{n}, v_{\delta t}^{n}\right)$ exists and is unique. Equation (10) allows to write $v_{\delta t}^{n+1}$ explicitly:

$$
v_{\delta t}^{n+1}=\frac{1}{1+\delta t}\left(\delta t F\left(\left|D u_{\delta t}^{n+1}\right|^{2}\right)+v_{\delta t}^{n}\right)
$$

and by induction we find

$$
0 \leq v_{\delta t}^{n+1} \leq\left(1-(1+\delta t)^{-(n+1)}\right) \mathrm{M}+(1+\delta t)^{-(n+1)}\left\|v_{0}\right\|_{L^{\infty}(\Omega)} .
$$

We deduce that $\left(v_{\delta t}^{n}\right)$ is uniformly bounded in $L^{\infty}(\Omega)$ and satisfies:

$$
0 \leq v_{\delta t}^{n} \leq \max \left(\mathrm{M},\left\|v_{0}\right\|_{L^{\infty}(\Omega)}\right):=\mathrm{M}^{\prime}, \text { for all } n \text { and } \delta t
$$



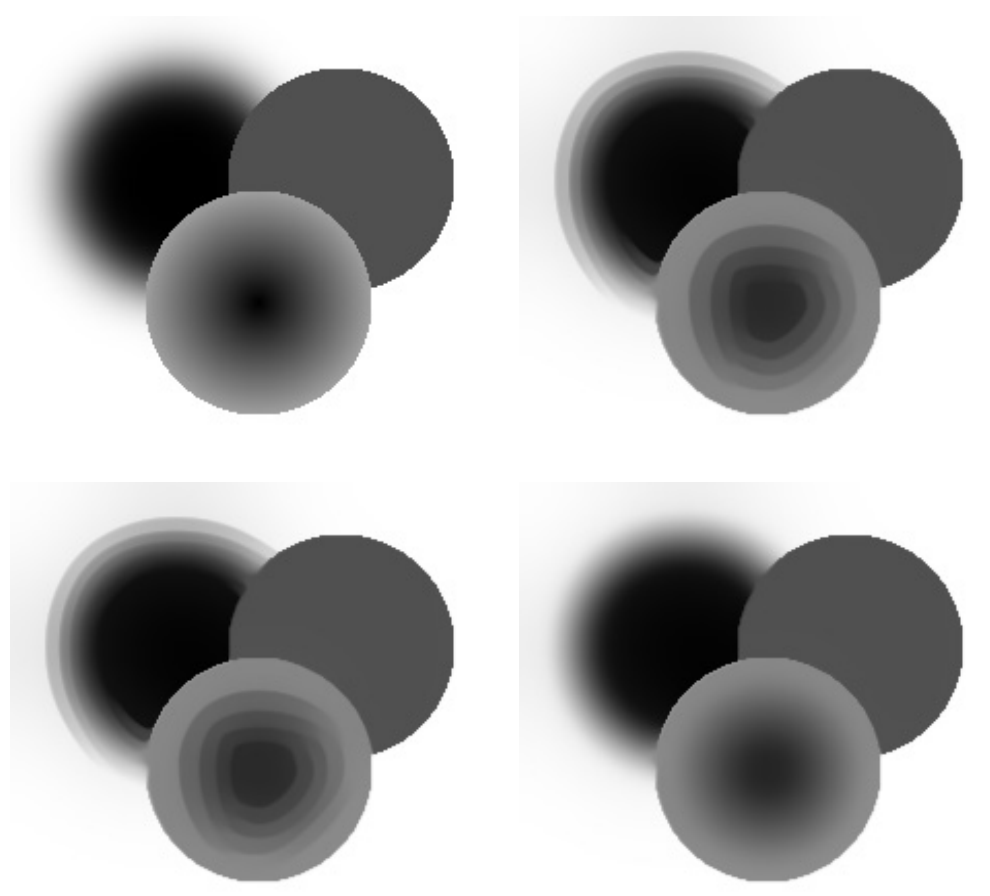

Figure 2. Top left: (a) Original image. Top right: (b) Image (a) filtered by the Perona-Malik model at scale 20. Bottom left: (c) Image (a) filtered by our scheme with $w=1$ at scale 20 . Bottom right: (d) Image (a) filtered by our scheme with $w=0.01$ at scale 20.

Using the fact that $g$ is a positive non-increasing function, we have $0<g\left(\mathrm{M}^{\prime}\right) \leq g\left(v_{\delta t}^{n}\right) \leq 1$. Therefore equation (9) is strictly elliptic and we know that there exists a unique solution $u_{\delta t}^{n+1}$ in $H^{1}(\Omega)$. In addition, $u_{\delta t}^{n+1}$ is given by the problem

$$
\min \left\{E_{(\delta t, n)}(w)=\int_{\Omega} g\left(v_{\delta t}^{n}\right)|D w|^{2} \mathrm{~d} x+\frac{1}{2 \delta t} \int_{\Omega}\left|w-u_{\delta t}^{n}\right|^{2} \mathrm{~d} x: w \in H^{1}(\Omega)\right\} .
$$

By the maximum principle, it is clear that for almost all $x \in \Omega$ we have

$$
\inf u_{0} \leq \cdots \leq \inf u_{\delta t}^{n} \leq u_{\delta t}^{n+1}(x) \leq \sup u_{\delta t}^{n} \leq \cdots \leq \sup u_{0}
$$

Multiplying by $u_{\delta t}^{n+1}$ the equation (9) and integrating on $\Omega$ we get

$$
0 \leq \delta t \int_{\Omega} g\left(v_{\delta t}^{n}\right)\left|D u_{\delta t}^{n+1}\right|^{2} \mathrm{~d} x \leq \int_{\Omega} u_{\delta t}^{n} u_{\delta t}^{n+1} \mathrm{~d} x-\int_{\Omega}\left|u_{\delta t}^{n+1}\right|^{2},
$$

from which we deduce

$$
\left\|u_{\delta t}^{n+1}\right\|_{L^{2}(\Omega)} \leq\left\|u_{\delta t}^{n}\right\|_{L^{2}(\Omega)} \leq \cdots \leq\left\|u_{0}\right\|_{L^{2}(\Omega)} .
$$

We now define the piecewise affine (in $t>0$ )

$$
\widehat{u}_{\delta t}(x, t)=(1-\theta) u_{\delta t}^{[t / \delta t]}(x)+\theta u_{\delta t}^{[t / \delta t]+1}(x)
$$



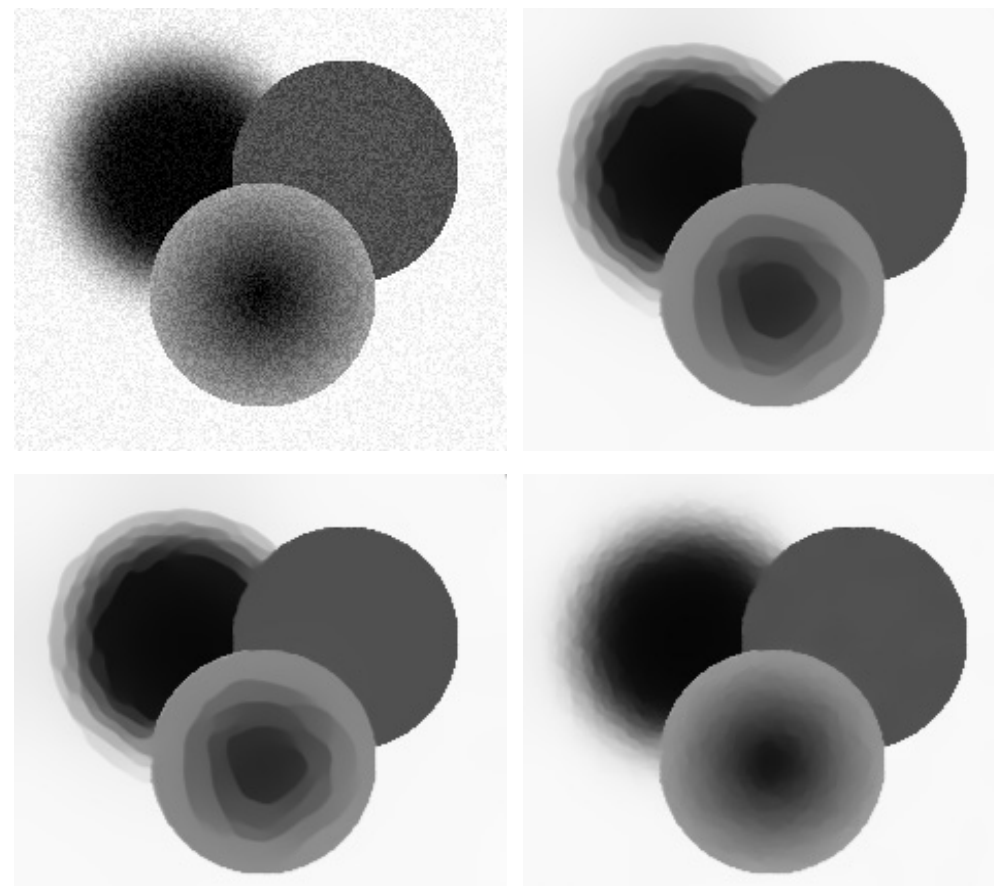

Figure 3. Same configuration as in Figure 2, with an additional noise.
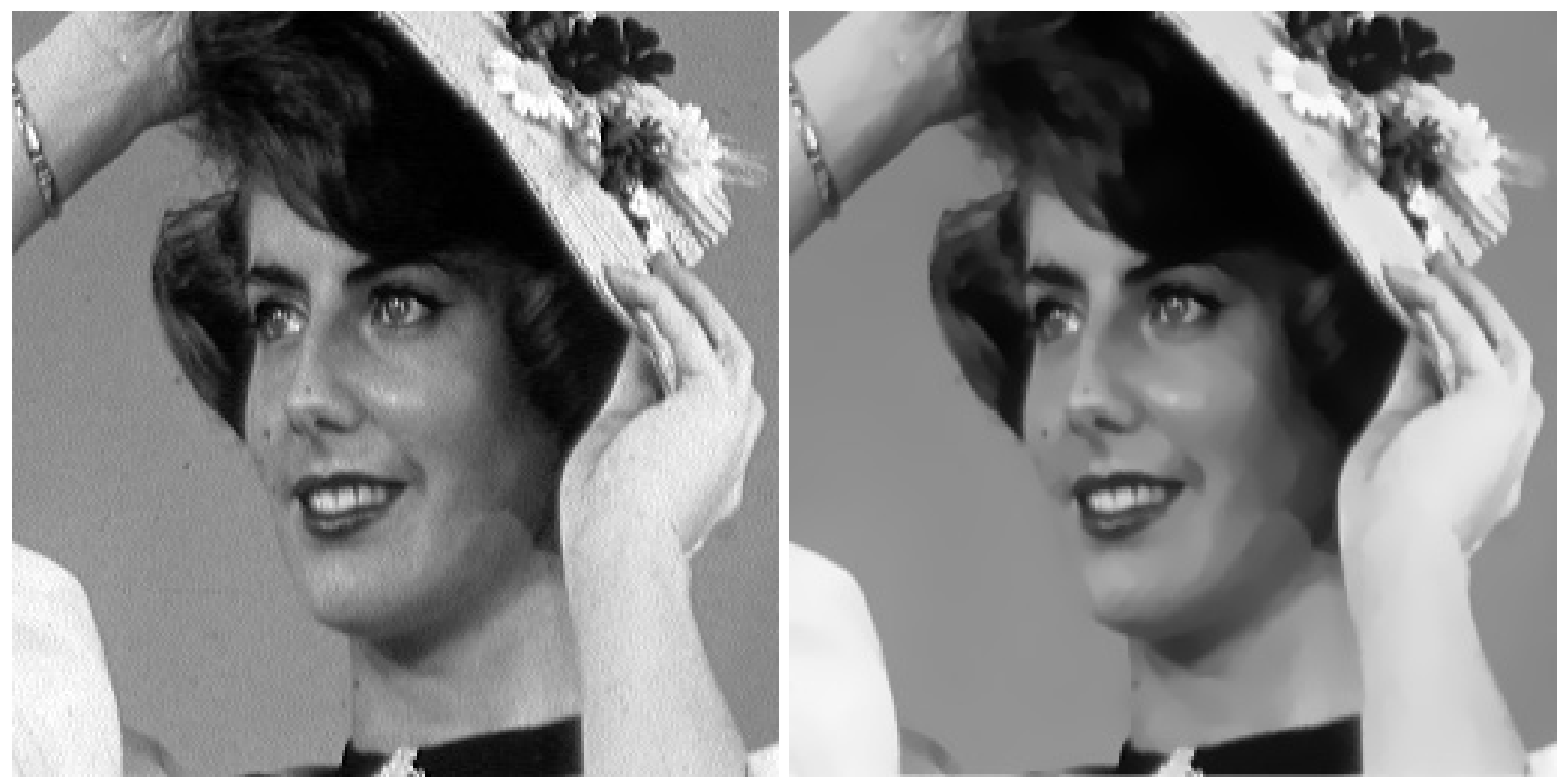

FIgURE 4. Right: original natural image. Left: the output of our scheme at scale 5. 
with $\theta=t / \delta t-[t / \delta t] \in[0,1)$. We also define $\left(\widehat{v}_{\delta t}\right)$ in the same way. Then we can write discrete system (11)-(12) in the form

$$
\begin{aligned}
\frac{\partial \widehat{u}_{\delta t}}{\partial t} & =\operatorname{div}\left(g\left(\tau^{-\delta t} v_{\delta t}\right) D u_{\delta t}\right) \\
\frac{\partial \widehat{v}_{\delta t}}{\partial t} & =F\left(\left|D u_{\delta t}\right|^{2}\right)-v_{\delta t}
\end{aligned}
$$

Lemma 2. $\left(\partial \widehat{v}_{\delta t} / \partial t\right)$ is uniformly bounded in $L^{\infty}((0, T) \times \Omega)$, and in particular

$$
\lim _{\delta t \rightarrow 0}\left\|\widehat{v}_{\delta t}-v_{\delta t}\right\|_{L^{\infty}\left((0, T) ; L^{2}(\Omega)\right)}=0 .
$$

Proof. From (27) and the inequalities (21), we easily deduce a uniform bound for $\left(\partial \widehat{v}_{\delta t} / \partial t\right)$,

$$
\left\|\frac{\partial \widehat{v}_{\delta t}}{\partial t}\right\|_{L^{\infty}((0, T) \times \Omega)} \leq \mathrm{M}^{\prime} .
$$

Let $t \in(0, T)$. We set $\theta=t / \delta t-[t / \delta t]$, and write

$$
\int_{\Omega}\left|\widehat{v}_{\delta t}(x, t)-v_{\delta t}(x, t)\right|^{2} \mathrm{~d} x=\int_{\Omega}\left|(1-\theta)\left(v_{\delta t}^{[t / \delta t]}(x)-v_{\delta t}^{[t / \delta t]+1}(x)\right)\right|^{2} \mathrm{~d} x .
$$

Hence

$$
\int_{\Omega}\left|\widehat{v}_{\delta t}(x, t)-v_{\delta t}(x, t)\right|^{2} \mathrm{~d} x \leq \delta t^{2} \int_{\Omega}\left|\frac{\partial \widehat{v}_{\delta t}}{\partial t}(x, t)\right|^{2} \mathrm{~d} x \leq \delta t^{2} \mathrm{M}^{\prime 2}
$$

(28) follows.

Lemma 3. $\left(u_{\delta t}\right)$ is uniformly bounded in $L^{\infty}\left(0, T ; H^{1}(\Omega)\right)$. More precisely we have:

$$
\left\|D u_{\delta t}\right\|_{L^{\infty}\left(0, T ; L^{2}(\Omega)\right)}^{2} \leq \frac{1}{g\left(M^{\prime}\right)}\left(\left\|D u_{0}\right\|_{L^{2}(\Omega)}^{2}+\frac{C}{g\left(M^{\prime}\right)}\left\|u_{0}\right\|_{L^{2}(\Omega)}^{2}\right)
$$

with $C=\left(\sup \left|g^{\prime}\right|\right)\left\|\frac{\partial \widehat{v}_{\delta t}}{\partial t}\right\|_{L^{\infty}((0, T) \times \Omega)}$.

Proof. First we establish a uniform bound for $\left(u_{\delta t}\right)$ in $L^{2}\left(0, T ; H^{1}(\Omega)\right)$, and prove the lemma by showing the following inequality:

$$
\left\|D u_{\delta t}\right\|_{L^{\infty}\left(0, T ; L^{2}(\Omega)\right)}^{2} \leq C_{1}\left\|D u_{0}\right\|_{L^{2}(\Omega)}^{2}+C_{2}\left\|D u_{\delta t}\right\|_{L^{2}\left(0, T ; L^{2}(\Omega)\right)}^{2}
$$

where $C_{1}, C_{2}>0$ are constants that will be made precise.

Multiplying (9) by $u_{\delta t}^{n+1}$ and integrating by part in $\Omega$ as in (24), we obtain

$$
\begin{aligned}
g\left(\mathrm{M}^{\prime}\right) \int_{n \delta t}^{(n+1) \delta t} \int_{\Omega}\left|D u_{\delta t}\right|^{2} \mathrm{~d} x \mathrm{~d} t & =g\left(\mathrm{M}^{\prime}\right) \delta t \int_{\Omega}\left|D u_{\delta t}^{n+1}\right|^{2} \mathrm{~d} x \\
& \leq \delta t \int_{\Omega} g\left(v_{\delta t}^{n}\right)\left|D u_{\delta t}^{n+1}\right|^{2} \mathrm{~d} x \\
& \leq\left\|u_{\delta t}^{n}\right\|_{L^{2}(\Omega)}^{2}-\left\|u_{\delta t}^{n+1}\right\|_{L^{2}(\Omega)}^{2}
\end{aligned}
$$


Then we deduce (for simplicity we use the notation $k:=[T / \delta t]$ )

$$
\begin{aligned}
g\left(\mathrm{M}^{\prime}\right) \int_{0}^{T} \int_{\Omega}\left|D u_{\delta t}\right|^{2} \mathrm{~d} x \mathrm{~d} t & =g\left(\mathrm{M}^{\prime}\right) \int_{0}^{\delta t k} \int_{\Omega}\left|D u_{\delta t}\right|^{2} \mathrm{~d} x \mathrm{~d} t+g\left(\mathrm{M}^{\prime}\right) \int_{\delta t k}^{T} \int_{\Omega}\left|D u_{\delta t}\right|^{2} \mathrm{~d} x \mathrm{~d} t \\
& \leq\left(\left\|u_{0}\right\|_{L^{2}(\Omega)}^{2}-\left\|u_{\delta t}^{k}\right\|_{L^{2}(\Omega)}^{2}\right)+\frac{T-\delta t k}{\delta t}\left(\left\|u_{\delta t}^{k}\right\|_{L^{2}(\Omega)}^{2}-\left\|u_{\delta t}^{k+1}\right\|_{L^{2}(\Omega)}^{2}\right) \\
& \leq\left\|u_{0}\right\|_{L^{2}(\Omega)}^{2}+\frac{T-\delta t(k+1)}{\delta t}\left\|u_{\delta t}^{k}\right\|_{L^{2}(\Omega)}^{2}-\frac{T-\delta t k}{\delta t}\left\|u_{\delta t}^{k+1}\right\|_{L^{2}(\Omega)}^{2}
\end{aligned}
$$

and since $\delta t k \leq T \leq \delta t(k+1)$, we obtain

$$
\int_{0}^{T} \int_{\Omega}\left|D u_{\delta t}\right|^{2} \mathrm{~d} x \mathrm{~d} t \leq \frac{1}{g\left(\mathrm{M}^{\prime}\right)}\left\|u_{0}\right\|_{L^{2}(\Omega)}^{2}
$$

This proves that $\left(u_{\delta t}\right)$ is uniformly bounded in $L^{2}\left(0, T ; H^{1}(\Omega)\right)$.

Now we show (31). For all $n \geq 0$, we have

$$
\begin{aligned}
& \int_{\Omega} g\left(v_{\delta t}^{n+1}\right)\left|D u_{\delta t}^{n+1}\right|^{2} \mathrm{~d} x-\int_{\Omega} g\left(v_{\delta t}^{n}\right)\left|D u_{\delta t}^{n}\right|^{2} \mathrm{~d} x= \\
& \qquad \int_{\Omega}\left(g\left(v_{\delta t}^{n+1}\right)-g\left(v_{\delta t}^{n}\right)\right)\left|D u_{\delta t}^{n+1}\right|^{2} \mathrm{~d} x+\int_{\Omega} g\left(v_{\delta t}^{n}\right)\left(\left|D u_{\delta t}^{n+1}\right|^{2}-\left|D u_{\delta t}^{n}\right|^{2}\right) \mathrm{d} x
\end{aligned}
$$

Using the minimum problem $(22)$ we have $E_{(\delta t, n)}\left(u_{\delta t}^{n+1}\right) \leq E_{(\delta t, n)}\left(u_{\delta t}^{n}\right)$, that is,

$$
\int_{\Omega} g\left(v_{\delta t}^{n}\right)\left|D u_{\delta t}^{n+1}\right|^{2} \mathrm{~d} x+\frac{1}{2 \delta t} \int_{\Omega}\left|u_{\delta t}^{n+1}-u_{\delta t}^{n}\right|^{2} \mathrm{~d} x \leq \int_{\Omega} g\left(v_{\delta t}^{n}\right)\left|D u_{\delta t}^{n}\right|^{2} \mathrm{~d} x .
$$

Then the second integral of (33) satisfies

$$
\int_{\Omega} g\left(v_{\delta t}^{n}\right)\left(\left|D u_{\delta t}^{n+1}\right|^{2}-\left|D u_{\delta t}^{n}\right|^{2}\right) \mathrm{d} x \leq 0
$$

The first integral of (33) we can be written

$$
\begin{aligned}
\int_{\Omega}\left(g\left(v_{\delta t}^{n+1}\right)-g\left(v_{\delta t}^{n}\right)\right)\left|D u_{\delta t}^{n+1}\right|^{2} \mathrm{~d} x & \leq \delta t \int_{\Omega}\left|\frac{g\left(v_{\delta t}^{n+1}\right)-g\left(v_{\delta t}^{n}\right)}{\delta t}\right|\left|D u_{\delta t}^{n+1}\right|^{2} \mathrm{~d} x \\
& \leq C \delta t \int_{\Omega}\left|D u_{\delta t}^{n+1}\right|^{2} \mathrm{~d} x,
\end{aligned}
$$

with $C:=\left(\sup \left|g^{\prime}\right|\right)\left\|\frac{\partial \widehat{v}_{\delta t}}{\partial t}\right\|_{L^{\infty}((0, T) \times \Omega)}$. Using this estimate in $(33)$, we get

$$
\int_{\Omega} g\left(v_{\delta t}^{n+1}\right)\left|D u_{\delta t}^{n+1}\right|^{2} \mathrm{~d} x-\int_{\Omega} g\left(v_{\delta t}^{n}\right)\left|D u_{\delta t}^{n}\right|^{2} \mathrm{~d} x \leq C \delta t \int_{\Omega}\left|D u_{\delta t}^{n+1}\right|^{2} \mathrm{~d} x .
$$

Taking the sum as $n$ varies from 0 to $[t / \delta t]$ and using the fact that $u_{\delta t}(\cdot, t)=u_{\delta t}^{[t / \delta t]+1}$, we obtain

$$
\begin{aligned}
\int_{\Omega} g\left(v_{\delta t}^{[t / \delta t]+1}\right)\left|D u_{\delta t}^{[t / \delta t]+1}\right|^{2} \mathrm{~d} x-\int_{\Omega} g\left(v_{0}\right)\left|D u_{0}\right|^{2} \mathrm{~d} x & \leq C \sum_{n=0}^{[t / \delta t]} \delta t \int_{\Omega}\left|D u_{\delta t}^{n+1}\right|^{2} \mathrm{~d} x \\
& \leq C \int_{0}^{\delta t([t / \delta t]+1)} \int_{\Omega}\left|D u_{\delta t}\right|^{2} \mathrm{~d} x \mathrm{~d} t .
\end{aligned}
$$


We conclude that for all $t \in(0, T)$

$$
\int_{\Omega} g\left(v_{\delta t}(\cdot, t)\right)\left|D u_{\delta t}(\cdot, t)\right|^{2} \mathrm{~d} x \leq \int_{\Omega} g\left(v_{0}\right)\left|D u_{0}\right|^{2} \mathrm{~d} x+C \int_{0}^{T} \int_{\Omega}\left|D u_{\delta t}\right|^{2} \mathrm{~d} x \mathrm{~d} t .
$$

Since $g\left(\mathrm{M}^{\prime}\right) \leq g\left(v_{\delta t}\right) \leq 1$, we get

$$
\left\|D u_{\delta t}(\cdot, t)\right\|_{L^{2}(\Omega)}^{2} \leq \frac{1}{g\left(\mathrm{M}^{\prime}\right)}\left(\int_{\Omega}\left|D u_{0}\right|^{2} \mathrm{~d} x+C \int_{0}^{T} \int_{\Omega}\left|D u_{\delta t}\right|^{2} \mathrm{~d} x \mathrm{~d} t\right),
$$

this proves (31). To conclude the proof of the lemma we combine the last result with (32) to obtain (30).

Lemma 4. $\left(\widehat{u}_{\delta t}\right)$ is uniformly bounded in $H^{1}((0, T) \times \Omega)$ and satisfies the inequality

$$
\frac{1}{2}\left\|\frac{\partial \widehat{u}_{\delta t}}{\partial t}\right\|_{L^{2}((0, T) \times \Omega)}^{2} \leq\left\|D u_{0}\right\|_{L^{2}(\Omega)}^{2}+\frac{C}{g\left(M^{\prime}\right)}\left\|u_{0}\right\|_{L^{2}(\Omega)}^{2},
$$

where $C$ is the constant of Lemma 3. In particular we have

$$
\lim _{\delta t \rightarrow 0}\left\|\widehat{u}_{\delta t}-u_{\delta t}\right\|_{L^{2}\left((0, T) ; L^{2}(\Omega)\right)}=0 .
$$

Proof. First we rewrite the inequality (34) in the form

$$
\begin{aligned}
\int_{n \delta t}^{(n+1) \delta t}\left\|\frac{\partial \widehat{u}_{\delta t}}{\partial t}\right\|_{L^{2}(\Omega)}^{2} \mathrm{~d} t & =\delta t \int_{\Omega}\left|\frac{u_{\delta t}^{n+1}-u_{\delta t}^{n}}{\delta t}\right|^{2} \mathrm{~d} x \\
& \leq 2\left(\int_{\Omega} g\left(v_{\delta t}^{n}\right)\left|D u_{\delta t}^{n}\right|^{2} \mathrm{~d} x-\int_{\Omega} g\left(v_{\delta t}^{n}\right)\left|D u_{\delta t}^{n+1}\right|^{2} \mathrm{~d} x\right) .
\end{aligned}
$$

Let $k:=[T / \delta t]$; we obtain using the last inequality

$$
\begin{aligned}
\frac{1}{2} \int_{0}^{T}\left\|\frac{\partial \widehat{u}_{\delta t}}{\partial t}\right\|_{L^{2}(\Omega)}^{2} \mathrm{~d} t= & \frac{1}{2}\left(\sum_{n=0}^{k-1} \delta t\left\|\frac{u_{\delta t}^{n+1}-u_{\delta t}^{n}}{\delta t}\right\|_{L^{2}(\Omega)}^{2}+(T-\delta t k)\left\|\frac{u_{\delta t}^{k+1}-u_{\delta t}^{k}}{\delta t}\right\|_{L^{2}(\Omega)}^{2}\right) \\
\leq & \sum_{n=0}^{k-1}\left(\int_{\Omega} g\left(v_{\delta t}^{n}\right)\left|D u_{\delta t}^{n}\right|^{2} \mathrm{~d} x-\int_{\Omega} g\left(v_{\delta t}^{n}\right)\left|D u_{\delta t}^{n+1}\right|^{2} \mathrm{~d} x\right) \\
& +\frac{T-\delta t k}{\delta t}\left(\int_{\Omega} g\left(v_{\delta t}^{k}\right)\left|D u_{\delta t}^{k}\right|^{2} \mathrm{~d} x-g\left(v_{\delta t}^{k}\right)\left|D u_{\delta t}^{k+1}\right|^{2} \mathrm{~d} x\right) \\
\leq & \int_{\Omega} g\left(v_{0}\right)\left|D u_{0}\right|^{2} \mathrm{~d} x \\
& +\sum_{n=1}^{k-1}\left(\int_{\Omega} g\left(v_{\delta t}^{n}\right)\left|D u_{\delta t}^{n}\right|^{2} \mathrm{~d} x-\int_{\Omega} g\left(v_{\delta t}^{n-1}\right)\left|D u_{\delta t}^{n}\right|^{2} \mathrm{~d} x\right) \\
& +\left(\frac{T-\delta t k}{\delta t} \int_{\Omega} g\left(v_{\delta t}^{k}\right)\left|D u_{\delta t}^{k}\right|^{2} \mathrm{~d} x-\int_{\Omega} g\left(v_{\delta t}^{k-1}\right)\left|D u_{\delta t}^{k}\right|^{2} \mathrm{~d} x\right) \\
& -\frac{T-\delta t k}{\delta t} \int_{\Omega} g\left(v_{\delta t}^{k+1}\right)\left|D u_{\delta t}^{k+1}\right|^{2} \mathrm{~d} x
\end{aligned}
$$


Let us estimate the last four terms. Since $g \leq 1,(38)$ satisfies

$$
\int_{\Omega} g\left(v_{0}\right)\left|D u_{0}\right|^{2} \mathrm{~d} x \leq\left\|D u_{0}\right\|_{L^{2}(\Omega)}^{2} .
$$

For the term (39), we proceed as in (35)

$$
\sum_{n=1}^{k-1}\left(\int_{\Omega} g\left(v_{\delta t}^{n}\right)\left|D u_{\delta t}^{n}\right|^{2} \mathrm{~d} x-\int_{\Omega} g\left(v_{\delta t}^{n-1}\right)\left|D u_{\delta t}^{n}\right|^{2} \mathrm{~d} x\right) \leq C \int_{\delta t}^{\delta t(k-1)} \int_{\Omega}\left|D u_{\delta t}\right|^{2} \mathrm{~d} x \mathrm{~d} t .
$$

We use $T-\delta t k \leq \delta t$ to estimate the term (40),

$$
\left(\frac{T-\delta t k}{\delta t} \int_{\Omega} g\left(v_{\delta t}^{k}\right)\left|D u_{\delta t}^{k}\right|^{2} \mathrm{~d} x-\int_{\Omega} g\left(v_{\delta t}^{k-1}\right)\left|D u_{\delta t}^{k}\right|^{2} \mathrm{~d} x\right) \leq C \int_{\delta t(k-1)}^{\delta t k} \int_{\Omega}\left|D u_{\delta t}\right|^{2} \mathrm{~d} x \mathrm{~d} t
$$

and since $T-\delta t k \geq 0,(41)$ is non-positive. Thus

$$
\frac{1}{2} \int_{0}^{T}\left\|\frac{\partial \widehat{u}_{\delta t}}{\partial t}\right\|_{L^{2}(\Omega)}^{2} \mathrm{~d} t \leq\left\|D u_{0}\right\|_{L^{2}(\Omega)}^{2}+C \int_{0}^{T} \int_{\Omega}\left|D u_{\delta t}\right|^{2} \mathrm{~d} x \mathrm{~d} t
$$

To find (36), in the last inequality we put the right hand side of (32) in place of the integral term.

To show (37) we proceed as in (29), then we obtain after integrating on $(0, T)$

$$
\int_{0}^{T} \int_{\Omega}\left|\widehat{u}_{\delta t}(x, t)-u_{\delta t}(x, t)\right|^{2} \mathrm{~d} x \mathrm{~d} t \leq \delta t^{2} \int_{0}^{T} \int_{\Omega}\left|\frac{\partial \widehat{u}_{\delta t}}{\partial t}\right|^{2} \mathrm{~d} x \mathrm{~d} t
$$

which goes to 0 as $\delta t$ goes to 0 .

Now we will focus on the regularity and the convergence of the sequence $\left(v_{\delta t}\right)$. The idea is the following: the fact that $v_{\delta t}^{0} \in H^{1}(\Omega)$, allows us to establish a regularity on the second derivative of $u_{\delta t}^{1}$ which in his turn used to show that $v_{\delta t}^{1} \in H^{1}(\Omega)$, and so on. For this, we will use the classical theory of topological degree and standard regularity results for solutions of elliptic equations (see Gilbarg and Trudinger [9], Meyers [16]), to establish the following lemma.

Lemma 5. Let $w \in H^{1}(\Omega) \cap L^{\infty}\left(\Omega ; \mathbb{R}_{+}\right)$such that $0<\lambda \leq w(x)$ a.e. in $\Omega, f \in L^{2}(\Omega)$ and $u \in H^{1}(\Omega)$ the solution of the elliptic problem

$$
\operatorname{div}(w D u)=f,\left.\frac{\partial u}{\partial n}\right|_{\partial \Omega}=0, \quad f_{\Omega} u(x) \mathrm{d} x=0
$$

Then for all bounded continuous function $\psi \in C(] 0, \infty[, \mathbb{R})$ satisfying $|\psi(s)| \leq \lambda_{0}$ and $|s \psi(s)| \leq \lambda_{1}$ for all $s>0$, we have

$$
\left\|\psi(|D u|) D^{2} u\right\|_{L^{2}(\Omega)} \leq \lambda^{-1}\left(\lambda_{0}\|f\|_{L^{2}(\Omega)}+\lambda_{1}\|D w\|_{L^{2}(\Omega)}\right) .
$$

The proof of Lemma 5 is given in Section 6.

Remark 1. Since the divergence term of (9) has zero average, we have for all $\delta t>0$ and all $n f_{\Omega} u_{0}(x) \mathrm{d} x=$ $f_{\Omega} x_{\delta t}^{n}(x) \mathrm{d} x$. Thus, using the fact that our model is grey level shift invariant, we can assume that $u_{0}$ has zero average in $\Omega$ : it is not restrictive as we may always replace $u_{0}$ with $u_{0}-f_{\Omega} u_{0}(x) \mathrm{d} x$. This allows to have $f_{\Omega} \mu_{\delta t}^{n}(x) \mathrm{d} x=0$ for all $\delta t$ and all $n$.

Lemma 6. For all $n \geq 0$, we have $v_{\delta t}^{n} \in H^{1}(\Omega)$. 
Proof. We begin by proving that $v_{\delta t}^{1} \in H^{1}(\Omega)$. Since $v_{\delta t}^{1}$ is a linear combination of $F\left(\left|D u_{\delta t}^{1}\right|^{2}\right)$ and $v_{0} \in H^{1}(\Omega)$, it amounts to show that $F\left(\left|D u_{\delta t}^{1}\right|^{2}\right) \in H^{1}(\Omega)$. The first step is to determine the distributional derivative of $F\left(\left|D u_{\delta t}^{1}\right|^{2}\right)$. For simplicity we use the notation $u:=u_{\delta t}^{1}$.

By applying Lemma 5 to the equation (9) with $n=0$, we know (from the proof of the same lemma) that there exists $\epsilon \in(0,1)$ such that $D^{2} u \in L^{1+\epsilon}(\Omega)$, and since $u \in H^{1}(\Omega)$ in particular we have $u \in W^{2,1}(\Omega)$. Then, there exists a sequence of $C^{2}$ functions $\left(u_{n}\right)_{n}$ that strongly converges to $u$ in $W^{2,1}(\Omega)$ and satisfy $u_{n} \rightarrow u$, $D u_{n} \rightarrow D u$ a.e. in $\Omega$.

Let $\phi \in C_{0}^{\infty}(\Omega)$. Since $F$ is bounded and continuous we have $\left|F\left(\left|D u_{n}\right|^{2}\right) \partial_{i} \phi\right| \leq \mathrm{M}\left|\partial_{i} \phi\right|$ and $F\left(\left|D u_{n}\right|^{2}\right) \rightarrow$ $F\left(|D u|^{2}\right)$ a.e. in $\Omega$. Then by applying the Lebesgue theorem we obtain the convergence

$$
\int_{\Omega} F\left(\left|D u_{n}\right|^{2}\right) \partial_{i} \phi \mathrm{d} x \rightarrow \int_{\Omega} F\left(|D u|^{2}\right) \partial_{i} \phi \mathrm{d} x, \quad \text { as } n \rightarrow \infty .
$$

By the fact that $F^{\prime}(s)=0$ for large values of $s$, it's clear that $\left(F^{\prime}\left(\left|D u_{n}\right|^{2}\right) \partial_{j} u_{n}\right)_{n}$ is bounded in $L^{\infty}(\Omega)$. Then there exists a function $\xi \in L^{\infty}(\Omega)$ and a subsequence still denoted by $\left(F^{\prime}\left(\left|D u_{n}\right|^{2}\right) \partial_{j} u_{n}\right)_{n}$ such that $F^{\prime}\left(\left|D u_{n}\right|^{2}\right) \partial_{j} u_{n} \stackrel{\star}{\rightarrow} \xi$ in $L^{\infty}(\Omega)$. By using the continuity of $F^{\prime}$ we have $F^{\prime}\left(\left|D u_{n}\right|^{2}\right) \partial_{j} u_{n} \rightarrow F^{\prime}\left(|D u|^{2}\right) \partial_{j} u$ a.e. in $\Omega$. Then $\xi=F^{\prime}\left(|D u|^{2}\right) \partial_{j} u$. Combining the last weak convergence with the strong convergence $\partial_{i j} u_{n} \phi \rightarrow \partial_{i j} u \phi$ in $L^{1}(\Omega)$ (here we use $\left\|\partial_{i j} u_{n} \phi-\partial_{i j} u \phi\right\|_{L^{1}} \leq\|\phi\|_{L^{\infty}}\left\|\partial_{i j} u_{n}-\partial_{i j} u\right\|_{L^{1}} \rightarrow 0$ ), we obtain

$$
\int_{\Omega}-2 F^{\prime}\left(\left|D u_{n}\right|^{2}\right) \partial_{i j} u_{n} \partial_{j} u_{n} \phi \mathrm{d} x \rightarrow \int_{\Omega}-2 F^{\prime}\left(|D u|^{2}\right) \partial_{i j} u \partial_{j} u \phi \mathrm{d} x, \quad \text { as } n \rightarrow \infty .
$$

The fact that the two sequences in the left hand side of (44) and (45) are identical, proves that the distributional derivative of $F\left(|D u|^{2}\right)$ is given by $-2 F^{\prime}\left(|D u|^{2}\right) D^{2} u D u$.

The second step is to show that $D\left(F\left(|D u|^{2}\right)\right) \in L^{2}(\Omega)$. Indeed, we have $\left|F^{\prime}\left(|D u|^{2}\right) \partial_{i} u(1+|D u|)\right| \leq$ $C(\mathrm{M})$ a.e. in $\Omega$ with $C(\mathrm{M})=\left(\mathrm{M}^{\frac{1}{2}}+\mathrm{M}\right)$. Then we can write

$$
\left\|D\left(F\left(|D u|^{2}\right)\right)\right\|_{L^{2}(\Omega)} \leq C(\mathrm{M})\left\|\frac{D^{2} u}{1+|D u|}\right\|_{L^{2}(\Omega)} .
$$

Applying once more the Lemma 5 to the equation (9), with $n=0$ and $\psi(s)=1 /(1+s)$ to conclude that the right hand side of $(46)$ is bounded in $L^{2}(\Omega)$ by writing

$$
\left\|\frac{D^{2} u}{1+|D u|}\right\|_{L^{2}(\Omega)} \leq\left(g\left(M^{\prime}\right)\right)^{-1}\left(\left\|\frac{u-u_{0}}{\delta t}\right\|_{L^{2}(\Omega)}+\left\|D v_{0}\right\|_{L^{2}(\Omega)}\right) .
$$

We return to equation (20). Since $v_{0} \in H^{1}(\Omega)$, we deduce that $v_{\delta t}^{1} \in H^{1}(\Omega)$. By induction we conclude that $v_{\delta t}^{n} \in H^{1}(\Omega)$ for all $n>0$. This proves the lemma.

Lemma 7. The sequence $\left(v_{\delta t}\right)$ is uniformly bounded in $L^{\infty}\left(0, T ; H^{1}(\Omega)\right)$. In addition we have

$$
\left\|D v_{\delta t}(\cdot, t)\right\|_{L^{2}(\Omega)} \leq \mathrm{e}^{K t}\left\|D v_{0}\right\|_{L^{2}(\Omega)}+K \int_{0}^{t} \mathrm{e}^{K(t-s)}\left\|\frac{\partial \widehat{u}_{\delta t}}{\partial t}(\cdot, s)\right\|_{L^{2}(\Omega)} \mathrm{d} s
$$

with $K=\left(g\left(M^{\prime}\right)\right)^{-1}\left(M^{\frac{1}{2}}+M\right)$.

Proof. Deriving the equation (20) with $n=0$, and using the $L^{2}$ norm, we get

$$
\left\|D v_{\delta t}^{1}\right\|_{L^{2}(\Omega)} \leq \frac{\delta t}{1+\delta t}\left\|D\left(F\left(\left|D u_{\delta t}^{1}\right|^{2}\right)\right)\right\|_{L^{2}(\Omega)}+\frac{1}{1+\delta t}\left\|D v_{0}\right\|_{L^{2}(\Omega)} .
$$


Then using (46) and (47), we obtain

$$
\left\|D v_{\delta t}^{1}\right\|_{L^{2}(\Omega)} \leq \frac{K \delta t}{1+\delta t}\left\|\frac{u_{\delta t}^{1}-u_{0}}{\delta t}\right\|_{L^{2}(\Omega)}+\frac{1+K \delta t}{1+\delta t}\left\|D v_{0}\right\|_{L^{2}(\Omega)}
$$

with $K:=\left(g\left(M^{\prime}\right)\right)^{-1} C(\mathrm{M})$. We may prove in the same way that

$$
\left\|D v_{\delta t}^{n+1}\right\|_{L^{2}(\Omega)} \leq \frac{K \delta t}{1+\delta t}\left\|\frac{u_{\delta t}^{n+1}-u_{\delta t}^{n}}{\delta t}\right\|_{L^{2}(\Omega)}+\frac{1+K \delta t}{1+\delta t}\left\|D v_{\delta t}^{n}\right\|_{L^{2}(\Omega)}
$$

Then by induction we get for all $n$

$$
\left\|D v_{\delta t}^{n}\right\|_{L^{2}(\Omega)} \leq \frac{K}{1+\delta t} \sum_{j=1}^{n}\left\{\left(\frac{1+K \delta t}{1+\delta t}\right)^{n-j} \delta t\left\|\frac{u_{\delta t}^{j}-u_{\delta t}^{j-1}}{\delta t}\right\|_{L^{2}(\Omega)}\right\}+\left(\frac{1+K \delta t}{1+\delta t}\right)^{n}\left\|D v_{0}\right\|_{L^{2}(\Omega)} .
$$

Finally by using the inequality

we obtain for all $t \in(0, T)(n=[t / \delta t])$,

$$
\left(\frac{1+K \delta t}{1+\delta t}\right)^{n-j} \leq \mathrm{e}^{K \delta t(n-j)}
$$

$$
\left\|D v_{\delta t}(\cdot, t)\right\|_{L^{2}(\Omega)} \leq K \int_{0}^{t} \mathrm{e}^{K(t-s)}\left\|\frac{\partial \widehat{u}_{\delta t}}{\partial t}(\cdot, s)\right\|_{L^{2}(\Omega)} \mathrm{d} s+\mathrm{e}^{K t}\left\|D v_{0}\right\|_{L^{2}(\Omega)} .
$$

In particular we have

$$
\left\|D v_{\delta t}(\cdot, t)\right\|_{L^{2}(\Omega)} \leq K T \mathrm{e}^{K T}\left\|\frac{\partial \widehat{u}_{\delta t}}{\partial t}\right\|_{L^{2}\left((0, T) ; L^{2}(\Omega)\right)}+\mathrm{e}^{K T}\left\|D v_{0}\right\|_{L^{2}(\Omega)},
$$

which is bounded according to Lemma 4 .

Proof of Theorem 1. According to (23) and Lemmas 3 and 4 there exist two subsequences, $\left(u_{\delta t_{j}}\right)$ and $\left(\widehat{u}_{\delta t_{j}}\right)$, and a function $u \in H^{1}(\Omega \times(0, T)) \cap L^{\infty}(\Omega \times(0, T))$ such that

$$
\begin{aligned}
\widehat{u}_{\delta t_{j}}, u_{\delta t_{j}} \stackrel{j \rightarrow+\infty}{\longrightarrow} u & \text { strongly in } L^{2}(\Omega \times(0, T)), \\
\widehat{u}_{\delta t_{j}}, u_{\delta t_{j}} \stackrel{j \rightarrow+\infty}{\longrightarrow} u & \text { weakly in } L^{2}\left(0, T ; H^{1}(\Omega)\right), \\
\widehat{u}_{\delta t_{j}} \stackrel{j \rightarrow+\infty}{\longrightarrow} u & \text { weakly in } H^{1}(\Omega \times(0, T)) .
\end{aligned}
$$

We draw the same conclusion from (21) and Lemmas 2 and 7 if we replace $\left(u_{\delta t_{j}}, \widehat{u}_{\delta t_{j}}\right)$ with $\left(v_{\delta t_{j}}, \widehat{v}_{\delta t_{j}}\right)$ and $u$ with $v$. It remains to prove the strong convergence (13) and the fact that $(u, v)$ is a solution of a system (7)-(8).

Up to a subsequence, we can assume that $v_{\delta t_{j}} \rightarrow v$ a.e. in $\Omega \times(0, T)$. In addition, by using the fact that $\left(\partial \widehat{v}_{\delta t} / \partial t\right)$ is bounded in $L^{\infty}(\Omega \times(0, T))$, we deduce that $\tau^{-\delta t_{j}} v_{\delta t_{j}} \rightarrow v$ a.e. in $\Omega \times(0, T)$ and by continuity of $g$, we also obtain that $g\left(\tau^{-\delta t_{j}} v_{\delta t_{j}}\right) \rightarrow g(v)$ a.e. in $\Omega \times(0, T)$.

Let $\phi \in C^{\infty}(\bar{\Omega} \times(0, T))$. Multiplying the equation (26) by $\phi$ with $\delta t_{j}$ in place of $\delta t$, and integrating the result in $\Omega \times(0, T)$, we get

$$
\int_{0}^{T} \int_{\Omega} \frac{\partial \widehat{u}_{\delta t_{j}}}{\partial t} \phi \mathrm{d} x \mathrm{~d} t=-\int_{0}^{T} \int_{\Omega} g\left(\tau^{-\delta t_{j}} v_{\delta t_{j}}\right) D u_{\delta t_{j}} D \phi \mathrm{d} x \mathrm{~d} t .
$$

We study the limit of the two terms of the equality as $j$ goes to $+\infty$. By using the weak convergence $\partial \widehat{u}_{\delta t_{j}} / \partial t \rightarrow$ $\partial u / \partial t$ in $L^{2}(\Omega \times(0, T))$,

$$
\int_{0}^{T} \int_{\Omega} \frac{\partial \widehat{u}_{\delta t_{j}}}{\partial t} \phi \mathrm{d} x \mathrm{~d} t \stackrel{j \rightarrow+\infty}{\longrightarrow} \int_{0}^{T} \int_{\Omega} \frac{\partial u}{\partial t} \phi \mathrm{d} x \mathrm{~d} t .
$$


Combining the weak convergence of $D u_{\delta t_{j}}$ to $D u$ in $L^{2}\left((\Omega \times(0, T)) ; \mathbb{R}^{2}\right)$ with the strong convergence of $g\left(\tau^{-h_{j}} v_{\delta t_{j}}\right) D \phi$ to $g(v) D \phi$ in $\left.L^{2}((\Omega \times(0, T))) ; \mathbb{R}^{2}\right)$ (using Lebesgue's dominated convergence theorem), we deduce that

$$
\int_{0}^{T} \int_{\Omega} g\left(\tau^{-h_{j}} v_{\delta t_{j}}\right) D u_{\delta t_{j}} D \phi \mathrm{d} x \mathrm{~d} t \stackrel{j \rightarrow+\infty}{\longrightarrow} \int_{0}^{T} \int_{\Omega} g(v) D u D \phi \mathrm{d} x \mathrm{~d} t .
$$

Then we obtain

$$
\int_{0}^{T} \int_{\Omega} \frac{\partial u}{\partial t} \phi \mathrm{d} x \mathrm{~d} t=-\int_{0}^{T} \int_{\Omega} g(v) D u D \phi \mathrm{d} x \mathrm{~d} t
$$

which means that $u$ is a weak solution of (7).

Now, to prove the convergence (13), it remains to show that $D u_{\delta t_{j}}$ strongly converges to $D u$ in $L^{2}((\Omega \times$ $\left.(0, T))) ; \mathbb{R}^{2}\right)$. For this we write

$$
\begin{aligned}
\int_{0}^{T} \int_{\Omega} g\left(\tau^{-\delta t_{j}} v_{\delta t_{j}}\right)\left|D u_{\delta t_{j}}-D u\right|^{2} \mathrm{~d} x \mathrm{~d} t= & \int_{0}^{T} \int_{\Omega} g\left(\tau^{-\delta t_{j}} v_{\delta t_{j}}\right)\left|D u_{\delta t_{j}}\right|^{2} \mathrm{~d} x \mathrm{~d} t \\
& -2 \int_{0}^{T} \int_{\Omega} g\left(\tau^{-\delta t_{j}} v_{\delta t_{j}}\right)\left(D u_{\delta t_{j}} . D u\right) \mathrm{d} x \mathrm{~d} t \\
& +\int_{0}^{T} \int_{\Omega} g\left(\tau^{-\delta t_{j}} v_{\delta t_{j}}\right)|D u|^{2} \mathrm{~d} x \mathrm{~d} t \\
= & -\int_{0}^{T} \int_{\Omega} \frac{\partial \widehat{u}_{\delta t_{j}}}{\partial t} u_{\delta t_{j}} \mathrm{~d} x \mathrm{~d} t+2 \int_{0}^{T} \int_{\Omega} \frac{\partial \widehat{u}_{\delta t_{j}}}{\partial t} u \mathrm{~d} x \mathrm{~d} t \\
& +\int_{0}^{T} \int_{\Omega} g\left(\tau^{-\delta t_{j}} v_{\delta t_{j}}\right)|D u|^{2} \mathrm{~d} x \mathrm{~d} t .
\end{aligned}
$$

Let us study the convergence of each term of (49) as $j$ goes to $+\infty$. Taking into account that $\partial \widehat{u}_{\delta t_{j}} / \partial t$ converges weakly to $\partial u / \partial t$ and that $u_{\delta t_{j}}$ converges strongly to $u$, both in $L^{2}(\Omega \times(0, T))$, we obtain:

$$
\begin{gathered}
\int_{0}^{T} \int_{\Omega} \frac{\partial \widehat{u}_{\delta t_{j}}}{\partial t} u_{\delta t_{j}} \mathrm{~d} x \mathrm{~d} t \stackrel{j \rightarrow+\infty}{\longrightarrow} \int_{0}^{T} \int_{\Omega} \frac{\partial u}{\partial t} u \mathrm{~d} x \mathrm{~d} t, \\
\int_{0}^{T} \int_{\Omega} \frac{\partial \widehat{u}_{\delta t_{j}}}{\partial t} u \mathrm{~d} x \mathrm{~d} t \stackrel{j \rightarrow+\infty}{\longrightarrow} \int_{0}^{T} \int_{\Omega} \frac{\partial u}{\partial t} u \mathrm{~d} x \mathrm{~d} t .
\end{gathered}
$$

Using the fact that $g\left(\tau^{-\delta t_{j}} v_{\delta t_{j}}\right) \rightarrow g(v)$ a.e. and $g\left(\tau^{-\delta t_{j}} v_{\delta t_{j}}\right)|D u|^{2} \leq|D u|^{2}$, we deduce (by Lebesgue's theorem)

$$
\int_{0}^{T} \int_{\Omega} g\left(\tau^{-\delta t_{j}} v_{\delta t_{j}}\right)|D u|^{2} \mathrm{~d} x \mathrm{~d} t \stackrel{j \rightarrow+\infty}{\longrightarrow} \int_{0}^{T} \int_{\Omega} g(v)|D u|^{2} \mathrm{~d} x \mathrm{~d} t
$$

thus

$$
\lim _{j \rightarrow+\infty} \int_{0}^{T} \int_{\Omega} g\left(\tau^{-\delta t_{j}} v_{\delta t_{j}}\right)\left|D u_{\delta t_{j}}-D u\right|^{2} \mathrm{~d} x \mathrm{~d} t=\int_{0}^{T} \int_{\Omega} \frac{\partial u}{\partial t} u \mathrm{~d} x \mathrm{~d} t+\int_{0}^{T} \int_{\Omega} g(v)|D u|^{2} \mathrm{~d} x \mathrm{~d} t .
$$

By density of $C^{\infty}(\bar{\Omega} \times(0, T))$ in $H^{1}(\Omega \times(0, T))$ we substitute $\phi$ by $u$ in the equation (48), and get

$$
\int_{0}^{T} \int_{\Omega} \frac{\partial u}{\partial t} u \mathrm{~d} x \mathrm{~d} t=-\int_{0}^{T} \int_{\Omega} g(v)|D u|^{2} \mathrm{~d} x \mathrm{~d} t
$$


then

$$
g\left(\mathrm{M}^{\prime}\right) \lim _{j \rightarrow+\infty} \int_{0}^{T} \int_{\Omega}\left|D u_{\delta t_{j}}-D u\right|^{2} \mathrm{~d} x \mathrm{~d} t \leq \lim _{j \rightarrow+\infty} \int_{0}^{T} \int_{\Omega} g\left(\tau^{-\delta t_{j}} v_{\delta t_{j}}\right)\left|D u_{\delta t_{j}}-D u\right|^{2} \mathrm{~d} x \mathrm{~d} t=0
$$

this shows the strong convergence (13).

Finally, up to a subsequence we have that $D u_{\delta t_{j}} \rightarrow D u$ a.e. in $\Omega \times(0, T)$ and the continuity of $F$ allows us to obtain that $F\left(\left|D u_{\delta t_{j}}\right|^{2}\right) \rightarrow F\left(|D u|^{2}\right)$ a.e. in $\Omega \times(0, T)$. This proves that $v$ is a solution of $(8)$.

\section{Appendix}

Proof of Lemma 5. By a density argument there exists a sequence $\left(w_{n}\right)$ in $C^{\infty}(\bar{\Omega})$ that converges to $w$ in $H^{1}(\Omega)$. Using classical truncation and convolution arguments we can chose $\left(w_{n}\right)$ in $L^{\infty}\left(\Omega ; \mathbb{R}_{+}\right)$and which satisfy $\lambda \leq w_{n}$ for all $n \geq 0$. We deduce that the solution $u_{n}$ of the elliptic problem:

$$
\operatorname{div}\left(w_{n} D u_{n}\right)=f,\left.\quad \frac{\partial u_{n}}{\partial n}\right|_{\partial \Omega}=0, \quad f_{\Omega} u_{n}(x) \mathrm{d} x=0
$$

exists and unique in $H^{1}(\Omega)$. Moreover, from $\left\|D w_{n}\right\|_{L^{\infty}(\Omega)}<\infty, f \in L^{2}(\Omega)$ and using classical regularity results it appears that $u_{n} \in H^{2}(\Omega)$.

First we prove that the inequality (43) is true for the regularized problem (50). For this writing (50) in the form

$$
w_{n} \Delta u_{n}=-f+D w_{n} D u_{n}
$$

and multiplying the equation by $\psi\left(\left|D u_{n}\right|\right)$, then we get using the $L^{2}$-norm

$$
\left\|\psi\left(\left|D u_{n}\right|\right) w_{n} \Delta u_{n}\right\|_{L^{2}(\Omega)} \leq\left\|\psi\left(\left|D u_{n}\right|\right) f\right\|_{L^{2}(\Omega)}+\left\|\psi\left(\left|D u_{n}\right|\right) D w_{n} D u_{n}\right\|_{L^{2}(\Omega)} .
$$

As $0<\lambda \leq w_{n},|\psi| \leq \lambda_{0}$ and $|s \psi(s)| \leq \lambda_{1}$ together with the last inequality, we obtain

$$
\left\|\psi\left(\left|D u_{n}\right|\right) \Delta u_{n}\right\|_{L^{2}(\Omega)} \leq \lambda^{-1}\left(\lambda_{0}\|f\|_{L^{2}(\Omega)}+\lambda_{1}\left\|D w_{n}\right\|_{L^{2}(\Omega)}\right)
$$

To continue we need the following lemma that we prove in the end of this section (let us recall $\Omega$ is the plane square $\left.(0,1)^{2}\right)$ :

Lemma 8. Let $u \in H^{2}(\Omega)$ an $\Omega$-periodic function, then for all bounded continuous function $\psi \in C(] 0, \infty[, \mathbb{R})$, we have the equality

$$
\|\psi(|D u|) \Delta u\|_{L^{2}(\Omega)}=\left\|\psi(|D u|) D^{2} u\right\|_{L^{2}(\Omega)} .
$$

Now we extend $u_{n}$ on all $\mathbb{R}^{2}$ by symmetry and periodicity to a function $\tilde{u}_{n} 2 \Omega$-periodic. Then invoking Lemma 8 , we get

$$
\left\|\psi\left(\left|D \tilde{u}_{n}\right|\right) D^{2} \tilde{u}_{n}\right\|_{L^{2}(2 \Omega)}=\left\|\psi\left(\left|D \tilde{u}_{n}\right|\right) \Delta \tilde{u}_{n}\right\|_{L^{2}(2 \Omega)},
$$

and by symmetry of $\tilde{u}_{n}$ in $2 \Omega$, that

$$
\left\|\psi\left(\left|D u_{n}\right|\right) D^{2} u_{n}\right\|_{L^{2}(\Omega)}=\left\|\psi\left(\left|D u_{n}\right|\right) \Delta u_{n}\right\|_{L^{2}(\Omega)},
$$

that we use in (52) to obtain

$$
\left\|\psi\left(\left|D u_{n}\right|\right) D^{2} u_{n}\right\|_{L^{2}(\Omega)} \leq \lambda^{-1}\left(\lambda_{0}\|f\|_{L^{2}(\Omega)}+\lambda_{1}\left\|D w_{n}\right\|_{L^{2}(\Omega)}\right)
$$

This proves that the inequality (43) is true for the regularized problem (50). 
Inequality (53) implies that the sequence $\left(\psi\left(\left|D u_{n}\right|\right) D^{2} u_{n}\right)$ is bounded. Then there exists a subsequence (still denoted by $\left.\left(\psi\left(\left|D u_{n}\right|\right) D^{2} u_{n}\right)\right)$ weakly convergent in $L^{2}(\Omega)$. Let us assume that its weak limit is $\psi(|D u|) D^{2} u$. Then by applying Fatou's lemma we obtain

$$
\begin{aligned}
\left\|\psi(|D u|) D^{2} u\right\|_{L^{2}(\Omega)} & \leq \liminf _{n \rightarrow+\infty}\left\|\psi\left(\left|D u_{n}\right|\right) D^{2} u_{n}\right\|_{L^{2}(\Omega)} \\
& \leq \lambda^{-1} \liminf _{n \rightarrow+\infty}\left(\lambda_{0}\|f\|_{L^{2}(\Omega)}+\lambda_{1}\left\|D w_{n}\right\|_{L^{2}(\Omega)}\right), \\
& \leq \lambda^{-1}\left(\lambda_{0}\|f\|_{L^{2}(\Omega)}+\lambda_{1}\|D w\|_{L^{2}(\Omega)}\right),
\end{aligned}
$$

showing in particular (43).

Before proving that the weak limit of $\left(\psi\left(\left|D u_{n}\right|\right) D^{2} u_{n}\right)$ is $\psi(|D u|) D^{2} u$, let us show that up to a subsequence, $\left(u_{n}\right)$ is strongly convergent in $H^{1}(\Omega)$. Indeed, multiplying $\operatorname{div}\left(w_{n} D u_{n}\right)=f$ by $u_{n}$ and integrating the result on $\Omega$,

$$
\int_{\Omega} w_{n}\left|D u_{n}\right|^{2} \mathrm{~d} x=-\int_{\Omega} f u_{n} \mathrm{~d} x
$$

The left hand side satisfies

$$
\lambda\left\|D u_{n}\right\|_{L^{2}(\Omega)}^{2} \leq \int_{\Omega} w_{n}\left|D u_{n}\right|^{2} \mathrm{~d} x,
$$

and using the Hölder and Poincaré-Wirtinger inequalities, we get for the right hand side

$$
\int_{\Omega} f u_{n} \mathrm{~d} x \leq\|f\|_{L^{2}(\Omega)}\left\|u_{n}\right\|_{L^{2}(\Omega)} \leq C\|f\|_{L^{2}(\Omega)}\left\|D u_{n}\right\|_{L^{2}(\Omega)} .
$$

We deduce that $\left(u_{n}\right)$ is uniformly bounded in $H^{1}(\Omega)$ and we have

$$
\left\|D u_{n}\right\|_{L^{2}(\Omega)} \leq \frac{C}{\lambda}\|f\|_{L^{2}(\Omega)} .
$$

Then there exists $u \in H^{1}(\Omega)$ and a subsequence still denoted by $\left(u_{n}\right)$ that strongly converges to $u$ in $L^{2}(\Omega)$, weakly in $H^{1}(\Omega)$ and a.e. in $\Omega$. To prove the strong convergence $D u_{n} \rightarrow D u$ in $L^{2}(\Omega)$ we can write

$$
\int_{\Omega} w_{n}\left|D u_{n}-D u\right|^{2} \mathrm{~d} x=\int_{\Omega} w_{n}\left|D u_{n}\right|^{2} \mathrm{~d} x-2 \int_{\Omega} w_{n} D u_{n} D u \mathrm{~d} x+\int_{\Omega} w_{n}|D u|^{2} \mathrm{~d} x,
$$

and

$$
\begin{aligned}
& \int_{\Omega} w_{n}\left|D u_{n}\right|^{2} \mathrm{~d} x=-\int_{\Omega} f u_{n} \mathrm{~d} x \stackrel{n \rightarrow+\infty}{\longrightarrow}-\int_{\Omega} f u \mathrm{~d} x \\
& \int_{\Omega} w_{n}|D u|^{2} \mathrm{~d} x \stackrel{n \rightarrow+\infty}{\longrightarrow} \int_{\Omega} w|D u|^{2} \mathrm{~d} x=-\int_{\Omega} f u \mathrm{~d} x \\
&-2 \int_{\Omega} w_{n} D u_{n} D u \mathrm{~d} x=2 \int_{\Omega} f u \mathrm{~d} x
\end{aligned}
$$

thus

$$
\lambda \int_{\Omega}\left|D u_{n}-D u\right|^{2} \mathrm{~d} x \leq \int_{\Omega} w_{n}\left|D u_{n}-D u\right|^{2} \mathrm{~d} x \stackrel{n \rightarrow+\infty}{\longrightarrow} 0 .
$$

Now we are going to study the convergence of the sequences $\left(D^{2} u_{n}\right)$ and $\left(\psi\left(\left|D u_{n}\right|\right)\right)$ to prove that $\left(\psi\left(\left|D u_{n}\right|\right) D^{2} u_{n}\right) \longrightarrow \psi(|D u|) D^{2} u$ weakly in $L^{2}(\Omega)$. 
Denoting $\Omega_{1}=(-1,2) \times(-1,2)$ and choosing a function $\xi \in C_{c}^{\infty}\left(\mathbb{R}^{2}\right)$, such that $\Omega \subset \subset \operatorname{supp}(\xi) \subset \subset \Omega_{1}$, and satisfies

$$
\xi(x)= \begin{cases}1 & \text { if } x \in \Omega \\ 0 & \text { if } x \in \mathbb{R}^{2} / \Omega_{1}\end{cases}
$$

Remark that $\tilde{u}_{n} \xi \in W_{0}^{2,1+\epsilon}\left(\Omega_{1}\right)$ for all $\epsilon \in(0,1)$ ( $\epsilon$ fixed later). Then, according to Gilbarg-Trudinger ([9] Chap. 9, Cor. 10), there exists a constant $C(\epsilon)>0$ such that

$$
\left\|D^{2}\left(\tilde{u}_{n} \xi\right)\right\|_{L^{1+\epsilon}\left(\Omega_{1}\right)} \leq C(\epsilon)\left\|\Delta\left(\tilde{u}_{n} \xi\right)\right\|_{L^{1+\epsilon}\left(\Omega_{1}\right)} .
$$

It follows that

$$
\begin{aligned}
\left\|D^{2} u_{n}\right\|_{L^{1+\epsilon}(\Omega)} & \leq\left\|D^{2}\left(\tilde{u}_{n} \xi\right)\right\|_{L^{1+\epsilon}\left(\Omega_{1}\right)}, \\
& \leq C(\epsilon)\left\|\Delta\left(\tilde{u}_{n} \xi\right)\right\|_{L^{1+\epsilon}\left(\Omega_{1}\right)}, \\
& \leq C(\epsilon)\left\{\left\|\xi \Delta \tilde{u}_{n}\right\|_{L^{1+\epsilon}\left(\Omega_{1}\right)}+\left\|\tilde{u}_{n} \Delta \xi\right\|_{L^{1+\epsilon}\left(\Omega_{1}\right)}+2\left\|D \tilde{u}_{n} D \xi\right\|_{L^{1+\epsilon}\left(\Omega_{1}\right)}\right\} \\
& \leq C(\epsilon) C\left\{\left\|\Delta \tilde{u}_{n}\right\|_{L^{1+\epsilon}\left(\Omega_{1}\right)}+\left\|\tilde{u}_{n}\right\|_{L^{1+\epsilon}\left(\Omega_{1}\right)}+\left\|D \tilde{u}_{n}\right\|_{L^{1+\epsilon}\left(\Omega_{1}\right)}\right\}
\end{aligned}
$$

where $C:=\max \left(\|\xi\|_{\infty}, 2\|D \xi\|_{\infty},\|\Delta \xi\|_{\infty}\right)$. Thus

$$
\left\|D^{2} u_{n}\right\|_{L^{1+\epsilon}(\Omega)} \leq 9 C(\epsilon) C\left\{\left\|\Delta u_{n}\right\|_{L^{1+\epsilon}(\Omega)}+\left\|u_{n}\right\|_{L^{1+\epsilon}(\Omega)}+2\left\|D u_{n}\right\|_{L^{1+\epsilon}(\Omega)}\right\} .
$$

The two sequences $\left(\left\|u_{n}\right\|_{L^{1+\epsilon}(\Omega)}\right)$ and $\left(\left\|D u_{n}\right\|_{L^{1+\epsilon}(\Omega)}\right)$ are bounded according to (54) and (55). For the sequence $\left(\left\|\Delta u_{n}\right\|_{L^{1+\epsilon}(\Omega)}\right)$ we use (51), and write

$$
\left\|\Delta u_{n}\right\|_{L^{1+\epsilon}(\Omega)} \leq \lambda^{-1}\left(\|f\|_{L^{1+\epsilon}(\Omega)}+\left\|D v_{n} D u_{n}\right\|_{L^{1+\epsilon}(\Omega)}\right) .
$$

Then it remains to show that the sequence $\left(\left\|D w_{n} D u_{n}\right\|_{L^{1+\epsilon}(\Omega)}\right)$ is bounded. Remark that by the symmetry and the fact that $u_{n}$ satisfies the Neumann type boundary, the equation

$$
\tilde{f}=\operatorname{div}\left(\tilde{w}_{n} D \tilde{u}_{n}\right)
$$

still holds in $\Omega_{2}:=(-2,3) \times(-2,3)$ where the functions $\tilde{f}$ and $\tilde{w}_{n}$ are defined in the same way that $\tilde{u}_{n}$. Then according to Meyers' theorem [16], Theorem 2, there exists $p>2$, depending only on $\max \tilde{w} / \min \tilde{w} \leq\|w\|_{\infty} / \lambda$, such that for all $x \in \Omega_{2}$ and for all $R>0$ satisfying $B(x, R) \subset B(x, 2 R) \subset \Omega_{2}$, we have:

$$
\left\|D \tilde{u}_{n}\right\|_{L^{p}(B(x, R))} \leq C\left\{R^{2\left(\frac{1}{p}-\frac{1}{2}\right)-1}\left\|\tilde{u}_{n}\right\|_{L^{2}(B(x, 2 R))}+R^{2\left(\frac{1}{p}-\frac{1}{2}\right)+1}\|\tilde{f}\|_{L^{2}(B(x, 2 R))}\right\} .
$$

Choosing $x \in \Omega$ and $R$ such that $\Omega \subset B(x, R)$, for example $x=\left(\frac{1}{2}, \frac{1}{2}\right)$ and $R=\sqrt{2}$, we deduce that $\left(\left|D u_{n}\right|\right)$ is bounded in $L^{p}(\Omega)$.

Coming back to (57) and choosing $\epsilon=(p-2) /(p+2)$, then the sequence $\left(D w_{n} D u_{n}\right)$ is bounded in $L^{1+\epsilon}(\Omega)$ and consequently $\left(D^{2} u_{n}\right)$ is bounded in $L^{1+\epsilon}(\Omega)$. We conclude that up to extracting a subsequence

$$
D^{2} u_{n} \stackrel{n \rightarrow+\infty}{\longrightarrow} D^{2} u \quad \text { weakly in } L^{1+\epsilon}(\Omega)
$$

Consider $\phi \in C^{\infty}(\bar{\Omega})$. Since $D u_{n} \rightarrow D u$ a.e. in $\Omega$, the continuity and boundedness of $\psi$, we obtain using the Lebesgue theorem

$$
\psi\left(\left|D u_{n}\right|\right) \phi \stackrel{n \rightarrow+\infty}{\longrightarrow} \psi(|D u|) \phi \text { in } L^{q}(\Omega) \text { for all } q \in[1, \infty)
$$


Finally, choosing $q$ such that $1 /(1+\epsilon)+1 / q=1$ and combining the last strong convergence with the weak convergence (58), we obtain

$$
\int_{\Omega} \psi\left(\left|D u_{n}\right|\right) D^{2} u_{n} \phi \mathrm{d} x \stackrel{n \rightarrow+\infty}{\longrightarrow} \int_{\Omega} \psi(|D u|) D^{2} u \phi \mathrm{d} x .
$$

This conclude the proof of the lemma.

To prove Lemma 8 we need the following result which comes from the topological degree's theory, and is a particular case of [12], Lemma 2.10 (see also [10], Th. 6):

Lemma 9. Let $f \in C^{1}\left(\mathbb{R}^{2} ; \mathbb{R}^{2}\right)$ an $\Omega$-periodic function. Then for all $\psi \in C_{0}(] 0,+\infty[)$ we have

$$
\int_{\Omega} \psi(|f(x)|) J_{f}(x) \mathrm{d} x=0
$$

where $J_{f}(x)$ is the Jacobian of $f$ at point $x$, i.e. $J_{f}(x)=\operatorname{det}\left[\partial_{i} f^{j}(x)\right]$.

Proof of Lemma 8. Let $u \in C^{2}\left(\mathbb{R}^{2}\right)$ an $\Omega$-periodic function, $\psi \in C_{0}(] 0, \infty[; \mathbb{R})$. Using (59):

$$
\int_{\Omega} \psi(|D u|) J_{D u} \mathrm{~d} x \mathrm{~d} y=0
$$

that is,

This implies

$$
\int_{\Omega} \psi(|D u|) \partial_{x x} u \partial_{y y} u \mathrm{~d} x \mathrm{~d} y=\int_{\Omega} \psi(|D u|)\left(\partial_{x y} u\right)^{2} \mathrm{~d} x \mathrm{~d} y .
$$

$$
\int_{\Omega} \psi(|D u|)\left(\left(\partial_{x x} u\right)^{2}+\left(\partial_{y y} u\right)^{2}+2 \partial_{x x} u \partial_{y y} u\right) \mathrm{d} x \mathrm{~d} y=\int_{\Omega} \psi(|D u|)\left(\left(\partial_{x x} u\right)^{2}+\left(\partial_{y y} u\right)^{2}+2\left(\partial_{x y} u\right)^{2}\right) \mathrm{d} x \mathrm{~d} y
$$

that is,

$$
\int_{\Omega} \psi(|D u|)|\Delta u|^{2} \mathrm{~d} x \mathrm{~d} y=\int_{\Omega} \psi(|D u|)\left|D^{2} u\right|^{2} \mathrm{~d} x \mathrm{~d} y .
$$

Since $|D u|$ is bounded $\left(u \in C^{2}\left(\mathbb{R}^{2}\right)\right.$ and $\Omega$-periodic), it's clear that (60) remains true if $\psi$ is only bounded and continuous function from $\mathbb{R}^{+}$to $\mathbb{R}$.

Now we show that (60) is true for $u$ in $H^{2}(\Omega)$. Indeed, by density argument there exists a sequence $\left(w_{n}\right) \in$ $C^{2}\left(\mathbb{R}^{2}\right) \cap H^{2}(\Omega)$ that converges to $u$ in $H^{2}(\Omega)$. In particular we have $\Delta w_{n} \rightarrow \Delta u, D^{2} w_{n} \rightarrow D^{2} u$ et $D w_{n} \rightarrow D u$ in $L^{2}(\Omega)$. In addition, there exists a subsequence still denoted by $\left(w_{n}\right)$ such that $D w_{n} \rightarrow D u$ a.e. in $\Omega$.

Using the fact that $\psi$ is bounded, there exists a function $h \in L^{\infty}(\Omega)$ and a subsequence still denoted by $\left(w_{n}\right)$ such that $\psi^{2}\left(D w_{n}\right) \stackrel{\star}{\rightarrow} h$ in $L^{\infty}(\Omega)$. Combining this with the strong convergences $\left|\Delta w_{n}\right|^{2} \rightarrow|\Delta u|^{2}$ and $\left|D^{2} w_{n}\right|^{2} \rightarrow\left|D^{2} u\right|^{2}$ in $L^{1}(\Omega)$, then we obtain:

$$
\psi^{2}\left(D w_{n}\right)\left|\Delta w_{n}\right|^{2} \rightarrow h|\Delta u|^{2} \quad \text { and } \quad \psi^{2}\left(D w_{n}\right)\left|D^{2} w_{n}\right|^{2} \rightarrow h\left|D^{2} u\right|^{2} \quad \text { in } \quad L^{1}(\Omega) .
$$

Now using the fact that $D w_{n} \rightarrow D u$ strongly and a.e. in $\Omega$ and the continuity of $\psi$ we deduce that $h=\psi^{2}(D u)$. Finally, the sequence $\left(w_{n}\right)$ can be chosen $\Omega$-periodic (for example as a convolution of $u$ with a smooth kernel), then we obtain the desired result by applying the equality (60) to $w_{n}$ and passing to the limit.

Acknowledgements. The first author would like to thank Prof. G.H. Cottet for valuable conversations. This research was done while the second author was still with CEREMADE, Université de Paris Dauphine. 


\section{REFERENCES}

[1] L. Alvarez, F. Guichard, P.-L. Lions and J.-M. Morel, Axioms and fundamental equations of image processing. Arch. Rational Mech. Anal. 123 (1993) 199-257.

[2] A. Belahmidi, Équations aux dérivées partielles appliquées à la restauration et à l'agrandissement des images. Ph.D. thesis, CEREMADE, Université de Paris-Dauphine, Paris (2003). Available at http://tel.ccsd.cnrs.fr.

[3] J. Canny, A computational approach to edge detection. IEEE Trans. Pattern Anal. Mach. Intell. 8 (1986) 679-698.

[4] F. Catté, P.-L. Lions, J.-M. Morel and T. Coll, Image selective smoothing and edge detection by nonlinear diffusion. SIAM J. Numer. Anal. 29 (1992) 182-193.

[5] P.G. Ciarlet, Introduction à l'analyse numérique matricielle et à l'optimisation. Collection Mathématiques Appliquées pour la Maîtrise. Masson, Paris (1982).

[6] G.H. Cottet and M. El-Ayyadi, A volterra type model for image processing. IEEE Trans. Image Process. 7 (1998) $292-303$.

[7] S. Esedoḡlu, An analysis of the Perona-Malik scheme. Comm. Pure Appl. Math. 54 (2001) 1442-1487.

[8] Y. Giga and S. Goto, Motion of hypersurfaces and geometric equations. J. Math. Soc. Japan 44 (1992) 99-111.

[9] D. Gilbarg and N.S. Trudinger, Elliptic partial differential equations of second order. Classics in Mathematics. Springer-Verlag, Berlin (2001). Reprint of the 1998 edition.

[10] E. Heinz, An elementary analytic theory of the degree of mapping in n-dimensional space. J. Math. Mech. 8 (1959) $231-247$.

[11] K. Höllig and J.A. Nohel, A diffusion equation with a nonmonotone constitutive function, in Systems of nonlinear partial differential equations (Oxford, 1982), Reidel, Dordrecht. NATO Adv. Sci. Inst. Ser. C: Math. Phys. Sci. 111 (1983) 409-422.

[12] O. Kavian, Introduction à la théorie des points critiques et applications aux problèmes elliptiques, volume 13 of Mathématiques 83 Applications (Berlin). Springer-Verlag, Paris (1993).

[13] B. Kawohl and N. Kutev, Maximum and comparison principle for one-dimensional anisotropic diffusion. Math. Ann. 311 (1998) 107-123.

[14] S. Kichenassamy, The Perona-Malik paradox. SIAM J. Appl. Math. 57 (1997) 1328-1342.

[15] D. Marr and E. Hildreth, Theory of edge detection. Proc. Roy. Soc. London B. 207 (1980) 187-217.

[16] N.G. Meyers, An $L^{p}$ e-estimate for the gradient of solutions of second order elliptic divergence equations. Ann. Scuola Norm. Sup. Pisa 17 (1963) 189-206.

[17] M. Nitzberg and T. Shiota, Nonlinear image filtering with edge and corner enhancement. IEEE Trans. Pattern Anal. Mach. Intell. 14 (1992) 826-833.

[18] P. Perona and J. Malik, Scale-space and edge detection using anisotropic diffusion. IEEE Trans. Pattern Anal. Mach. Intell. 12 (1990) 629-639.

[19] R.T. Whitaker and S.M. Pizer, A multi-scale approach to nonuniform diffusion. CVGIP: Image Underst. 57 (1993) $99-110$.

[20] Y. You, W. Xu, A. Tannenbaum and M. Kaveh, Behavioral analysis of anisotropic diffusion in image processing. IEEE Trans. Image Process. 5 (1996) 1539-1553.

To access this journal online:

www.edpsciences.org 